\title{
Cooperation in a dynamic setting with asymmetric environmental valuation and responsibility
}

\author{
Francisco Cabo - Mabel Tidball
}

the date of receipt and acceptance should be inserted later

\begin{abstract}
We analyze an environmental agreement as a cooperative differential game between two regions. The agreement is jointly profitable, since the current contributions in terms of emissions reduction is overcompensated by the benefits of a cleaner environment now and in the future. The regions are asymmetric in two respects: the valuation of a cleaner environment and the responsibility for the state of the environment at the beginning of the agreement. Unlike standard dynamic distribution schemes, the proposed mechanism distributes the efforts of lowering current emissions, not the benefits of a cleaner environment. It is built following an axiomatic approach. It must be time consistent: at any intermediate time, no country can do better by deviating from cooperation. A benefits pay principle is also required: the greater one region's relative benefit from cooperation, the greater must be its relative contribution. A novelty of our approach is the addition of a responsibility or polluter pay principle: a region's relative contribution increases with its responsibility. We characterize a family of dynamic distribution schemes which satisfy the three desired axioms. Interestingly, the proposed scheme could equivalently arise from the Nash bargaining solution considering an asymmetric bargaining power.
\end{abstract}

Keywords Cooperative differential game - Dynamic distribution procedure - Time consistency .

Responsibility · Benefits pay principle · Asymmetric Nash Bargaining solution.

Acknowledgments We would like to thank Justin Leroux and Georges Zaccour for their useful comments and suggestions.

Francisco Cabo received fundings from the Spanish Government (projects ECO2014-52343-P and ECO2017-82227-P), and from Junta de Castilla y León (projects VA024P17 and VA105G18), co-

Francisco Cabo (corresponding author)

IMUVa, Universidad de Valladolid. Avda. Valle Esgueva, 6, 47011, Valladolid, Spain.

Tel.: +34-9836662 Fax: +34-9833299 E-mail: fjcabo@uva.es ORCID: 0000-0003-2958-9595

Mabel Tidball

CEE-M, Université de Montpellier, CNRS, INRAE, Institut Agro, Montpellier, France.

0000-0002-5576-0977 
financed by FEDER funds. Mabel Tidball received funding from the project GREEN-Econ (ANR16-CE03-0005) and the LabEx Entreprendre (ANR-10-LABX-11-01).

Conflicts of interest/Competing interests The authors have no conflicts of interest to declare that are relevant to the content of this article.

\section{Introduction}

In this paper we propose a dynamic distribution scheme which specifies how to share the effort that the cooperative parties in an environmental agreement need to undertake in order to mitigate an environmental problem. The sharing scheme focuses on a finite time environmental agreement and satisfies three desirable properties: time consistency, a benefits pay principle, and a polluter pay principle.

We start by considering a cooperative agreement to reduce emissions for a limited period in order to fight a common environmental problem, which is jointly beneficial for two regions. In particular, we analyze a stock pollution problem as, for example, two neighboring countries that share a lake polluted from wastewater discharges. An agreement to reduce discharges by the two countries across a finite period would improve the water quality in the future. The two regions bear immediate costs in terms of lower emissions and the subsequent losses in production, within the cooperative period. These losses are large in comparison with the slight immediate improvement of the water quality. However, the benefits from the agreement, in the form of lower damage from a less polluted environment, extend far beyond the cooperative period. The accumulated benefits from a cleaner environment outweigh current costs, which makes cooperation globally rational. These accumulated future benefits depend on the abatement efforts within cooperation, i.e. on the water quality at the end of the cooperative period. Additionally, we assume that the two countries value the environment differently and hence obtain different gains from cooperation. For example, one might be interested in fishing activities, while the other might assign the lake a recreational value. Other stock pollution problems characterized by immediate costs and future rewards are those agreements that seek to avoid an environmental catastrophe. The current costs of not crossing a catastrophic threshold are compensated by the future benefits of avoiding this catastrophe. Moreover, the damaging effects of the catastrophe do not need be the same for the different agents involved. ${ }^{1}$

Typically, different gains from cooperation are not the only source of asymmetry. In stock pollution problems it is commonly the case that the regions involved are responsible for the current state of the environmental problem in different ways. There is a wide range of literature which studies the different responsibilities of countries in the global warming problem. This discrepancy between the distribution of climate-change damages and responsibilities from past emissions is, for example, highlighted in De

\footnotetext{
1 The role of uncertainty in the context of climate agreements is analyzed by Barrett (2013).
} 
Villemeur and Leroux (2011). Several works analyze to what extent industrialized countries are more responsible than developing countries for the current carbon dioxide concentration in the atmosphere. ${ }^{2}$

The decomposition over time of the cooperative payoff from an environmental agreement when players are asymmetric is analyzed, for example, in Fanokoa et al. (2011) for a game of pollution control, or in Cabo et al. (2006) who also considers trade. Our proposal shares with these works the asymmetry in the benefits stemming from cooperation. Nonetheless, two characteristics separate our analysis from the existing literature. One is the asymmetry in the responsibility for past emissions. The other is the difficulty to redistribute the benefits from cooperation, because the benefits for a cleaner environment are region-specific and because they come at the end of cooperation to a large extent. Motivated by these two facts, our approach partially deviates from the standard approach to distribute payoffs in a cooperative dynamic game.

A dynamic environmental agreement can be regarded as a dynamic collective action problem where commitment is not available. Agents need to decide whether and to what extent they participate in the voluntary provision of a public good (environmental quality). The basic question in public good problems is how to alleviate the free-riding problem. In these collective action problem it is commonly agreed that when agents behave rationally, and take into consideration the strategic interaction, contribution decreases as agents anticipate that by an increase in his own contribution others will respond by reducing their contribution (Becket 1974). This is, for example, the case in Fershtman and Nitzan (1991) who analyze the collective action problem as an infinite horizon differential game. They show that under linear feedback strategies, when players base their behavior on the progress made in terms of collective contributions, the free-riding problem aggravates. On the other hand, Wirl (1996) revisits Fershtman and Nitzan and proves that non-linear strategies not only lead to higher individual contribution but they may even support (given appropriate initial conditions) a provision of public goods that exceeds the cooperative outcome. In the same vein Guttman (1978) and (1987) propose situations in this last direction. They consider a two-part contribution: a flat contribution, plus a matching rate of what all others cooperates. Under this specification it is possible that the rational behavior of the individuals leads to a higher contribution to the public good.

Our approach starts from the concept of an imputation distribution procedure (IDP), which defines a dynamic sharing rule for a cooperative differential game satisfying desirable properties. This central concept was introduced in Petrosyan and Danilov (1979) and later in Petrosyan (1993). The concept was first used in a cooperative game of pollution reduction in Petrosyan and Zaccour (2003). As explained

\footnotetext{
2 From 1850 to 2010, Ward and Mahowald (2014) estimate that the responsibility for a rise in temperature assigned
} to annex I countries can be around 58\%, (42\% for non-annex I). Similarly, from 1850 to 2005 , using the Community Earth System Model (CESM), Wei et al. (2016) estimate the responsibility for climatic change of developed countries to be between 53\%-61\%, and for developing countries to be approximately 39\%-47\%. Similarly, according to Zhang et al. (2008), between 1850 and 2004 the G8 countries accounted for $61 \%$ of GHG emissions. 
in Jørgensen and Zaccour (2001), Zaccour (2008), and Yeung and Petrosyan (2018), and the references therein, a dynamic distribution scheme seeks to distribute the gains from cooperation, between players and across time, following a particular solution concept such as the Shapley value, the egalitarian rule, or the Nash bargaining solution (NBS). We differ from this approach in two respects. First, the benefits from cooperation come in the form of a cleaner environment, which is differently enjoyed by the two countries. Although some of these benefits already take place withing cooperation, they are mainly realized once cooperation halts. The cooperative gains after cooperation cannot be distributed and our main focus instead is to distribute the effort that the agreement imposes on the cooperating agents. Second, we do not borrow a solution concept from the literature, but define a general distribution scheme which satisfies three desirable properties.

First, the agreement must be time consistent or internally stable, implying that at any moment within the cooperative period each region prefers to maintain cooperation rather than to deviate from the agreement and play non-cooperatively henceforth. The question of how to distribute the cooperative effort when the benefits emerge once cooperation halts was analyzed in Cabo and Tidball (2017), who proposed a time-consistent IDP. Based on this procedure, we define here an IDP which satisfies two additional properties. The analysis of joint projects without commitment also assumes that the cost in terms of contributions is borne instantaneously while the benefits from the agreement come at the end of the cooperation. The dynamic contribution to a joint project is analyzed in Admati and Perring (1991), who find that a game in which, at each stage, one of the players decides his contribution does not lead to an efficient outcome. By contrast, efficiency would arise for a subscription game in which players bear the cost only if the project is completed.

Second, given the asymmetry in the damage caused by pollution, a benefits pay principle (BPP) seems to be a desirable property. When analyzing how to share the cost of a public good, the benefit principle is probably the most well known equity condition. It states that everyone should pay for the public good according to his/her marginal willingness to pay. This principle dates back to Wicksell (1896/1958). In the literature on taxation this benefit principle states that the tax burdens should be related to the benefits received. ${ }^{3}$ In our formulation, the public good stems from the cooperative agreement in the form of a cleaner environment. The benefit principle states that at any time the relative effort that a region contributes to the agreement must be positively correlated to the relative benefit it will get from a less polluted environment.

Third, since regions are differently responsible for past emissions, we believe that the dynamic distribution scheme must satisfy a responsibility or polluter pay principle (PPP). To the best of our knowledge, the addition of a responsibility axiom to the definition of a dynamic scheme is new in the

\footnotetext{
3 Buchholz \& Peters (2007) show that the benefits principle in the case of public goods is equivalent to other equity concepts: Proportional contributions (cost share proportional to the marginal willingness to pay), and equality of sacrifice (every agent shares the same burden).
} 
literature. The polluter pay principle goes back at least to 1920 and it is normally implemented through two different policy approaches: command-and-control and market-based. Pigouvian taxes designed to correct externalities are commonly accepted by environmental economists in order to achieve efficiency (Pigou, 1920). Nevertheless, other social considerations such as equity, rights, or political considerations often play a role when defining policy instruments. Here we propose a distribution scheme which satisfies a responsibility principle. This principle which attempts to assign responsibility to a particular region for its past emissions has been discussed by Singer (2004). According to this principle, at any time within the cooperative period the relative effort that a region contributes to the agreement (from this instant on) must be positively correlated to its relative responsibility for all past emissions (i.e. its relative responsibility from the current state of the environment). Responsibility is defined following Hayner and Weisbach (2016) as the percentage of the initial stock of pollution a region is responsible for, minus the relative damage it bears from this pollution. ${ }^{4}$

We build a dynamic sharing rule in order to satisfy the three desired axioms. It defines the share of the global surplus allocated to one region, at each time, as a combination between the region's relative benefit from the agreement and its responsibility. It is worth noting that, although the proposed sharing rule is not based on a specific cooperative solution concept, it can be compared against the Nash bargaining Solution (NBS). Assuming that the region with the highest relative benefit from the agreement is, at the same time, the least responsible from the environmental problem then, the egalitarian rule or symmetric NBS does not satisfy the required axioms. More importantly, the proposed scheme is equivalent to a NBS with asymmetric bargaining power between the two regions. The region's bargaining power is equal to the share of the global surplus that our sharing procedure allocates to this region from a given time on.

Under the simplifying assumption that the damage from pollution within cooperation is negligible with respect to the damage beyond cooperation and if the damage function is multiplicatively separable into a region-specific parameter and a function of pollution, then the relative damage from pollution exactly matches the relative benefit from the agreement. Under this hypothesis, the greater the relative benefit, the greater the relative damage and hence the lower the responsibility. Thus, the total effect of a higher relative benefit from cooperation on contribution is described by a positive direct effect (from the BPP) plus a negative indirect effect from a lower responsibility (from the PPP). We characterize the condition under which the net effect is positive and a strong-BPP applies. Under these simplify-

\footnotetext{
4 It is important to state clear the difference between the definition of the benefit principle proposed and the commonly referred as the beneficiary-pays principle in the global warming literature. This latter refers to the responsibility of the countries who were benefited from the past emissions that generated the problem of climate change and their moral duty to address the associated damages. That is precisely what we refer to as the responsibility axiom or polluters pay principle.
} 
ing assumptions and considering a linear quadratic specification, a numerical example illustrates the proposed IDP. This example corroborates the theoretical results obtained.

In Section 2 we present the model which describes cooperation and what would be the noncooperative outside option. It also gives a definition of responsibility and defines the three desired axioms. Section 3 introduces the IDP that satisfies them and proves the equivalence with the asymmetric NBS. The procedure is applied to a numerical example in Section 4. The sensitivity with respect to the length of the cooperative time horizon is studied in Section 5. The conclusions are presented in Section 6 .

\section{The model}

We analyze a cooperative environmental agreement within a finite period $[0, T]$, by which players reduce their flow of emissions (and current gains from production), in exchange for a greater joint gain in terms of a cleaner environment, now and specially in the future.

\subsection{Cooperative solution and non cooperative equilibria}

We consider two different regions, 1 and 2, whose productive activities at time $\tau$ require the emission of flows of pollutants, $E^{i}(\tau), i \in\{1,2\}$. The emissions in both regions give rise to a pollution stock according to the dynamics equation: ${ }^{5}$

$$
\dot{P}(\tau)=F\left(E^{1}(\tau), E^{2}(\tau), P(\tau)\right), \quad P(0)=P_{0}
$$

where the evolution of the pollution stock is described by a differentiable function, $F$, increasing in emissions and decreasing in the current state of pollution, according to the assimilative capacity of the environment; and where $P_{0}$ is the initial pollution stock.

Any other input or technology accumulation process is ignored, and hence gains from production are fully determined by current emissions. Moreover, pollution causes an environmental damage. Thus, the flow of payoffs for region $i$ can be written as a differentiable and additively separable function of the gains from production associated with this region emissions, $g^{i}\left(E^{i}(\tau)\right)$, and the damage for this region generated by the pollution stock, ${ }^{6} l^{i}(P(\tau))$ :

$$
w^{i}\left(E^{i}(\tau), P(\tau)\right)=g^{i}\left(E^{i}(\tau)\right)-l^{i}(P(\tau))
$$

\footnotetext{
5 A superscript in a given variable refers to the specific region. Variables without superscript indicate global quantities for the two regions jointly considered.

6 Notice that although we refer to gains from emissions and losses associated with the pollution stock, our analysis would be equally valid under a more general formulation in which gains are also made dependent on the stock, $g^{i}\left(E^{i}(\tau), P(\tau)\right)$ (if, for example, productivity is negatively affected by the pollution stock), and losses also depend on the flow of emissions, $l^{i}\left(E^{i}(\tau), P(\tau)\right)$ (if emissions also cause environmental damages).
} 
At time 0 the two regions agree to cooperate for a finite period $[0, T]$. We do not model how the regions behave from this moment $T$ on. They could renegotiate the cooperative agreement, with the same or a different length, behave non-cooperatively, or many other alternatives. In either case, the lasting effects of pollution beyond the cooperative period, can be defined as dependent on the pollution stock at time $T$. The greater the pollution stock at $T$, the higher the damage caused by pollution initially, and from $T$ onward. This is collected by a upward sloping and convex scrap value, $D^{i}(P(T))$.

If the two regions agree to cooperate, they have to determine optimal emissions in order to maximize joint welfare:

$$
\max _{E^{i}(\tau), i \in\{1,2\}} \sum_{i=1}^{2}\left\{\int_{0}^{T} w^{i}\left(E^{i}(\tau), P(\tau)\right) e^{-\rho \tau} d \tau-D^{i}(P(T)) e^{-\rho T}\right\},
$$

subject to the pollution stock dynamics in (1), and where $\rho$ represents the instantaneous discount rate. Define by $E_{\mathrm{C}}^{i}(\tau)$ and $P_{\mathrm{C}}(\tau)$ the cooperative emissions of region $i$ and the pollution stock under the optimal cooperative solution at time $\tau \in[0, T]$. For shortness, denote $w_{\mathrm{C}}^{i}(\tau)=w^{i}\left(E_{\mathrm{C}}^{i}(\tau), P_{\mathrm{C}}(\tau)\right)$, $g_{\mathrm{C}}^{i}(\tau)=g^{i}\left(E_{\mathrm{C}}^{i}(\tau)\right)$ and $l_{\mathrm{C}}^{i}(\tau)=l^{i}\left(P_{\mathrm{C}}(\tau)\right)$ the optimal instantaneous welfare, gains from emissions and losses from pollution of region $i$ at time $\tau \in[0, T]$.

We do not presuppose a sharing mechanism between the two agents and across time. This will be the main aim of the analysis. We neither assume the existence of a commitment device that renders the agreement binding. Hence, at any revision time $t \in(0, T)$ agents might deviate and move to a noncooperative mode of play. As is common in the literature, we assume that once cooperation is halted, the two regions play non-cooperatively henceforth, at least until time $T$. Alternatively, one could argue that they could, for example, decide to renegotiate an agreement at any time after $t$ and prior to $T$. Such a possibility is introduced by Sorger (2006) who proposed an immediate renegotiation when the agreement is broken. ${ }^{7}$

In the non-cooperative mode of play starting at time $t$, each region $i \in\{1,2\}$ solves the maximization problem:

$$
\max _{E^{i}(\tau)} \int_{t}^{T} w^{i}\left(E^{i}(\tau), P(\tau)\right) e^{-\rho(\tau-t)} d \tau-D^{i}(P(T)) e^{-\rho(T-t)},
$$

subject to the stock pollution dynamics in (1) but with $P(t)=P_{\mathrm{C}}(t)$. Considering a feedback information structure, non-cooperative regions choose emissions strategies dependent on the stock of pollution as well as on calendar time. Let $E_{\mathrm{N}}^{i}(\tau ; t)$ and $P_{\mathrm{N}}(\tau ; t)$ denote the optimal emissions and pollution stock in the feedback Nash equilibrium of the non-cooperative game starting at time $t$. For shortness, denote $w_{\mathrm{N}}^{i}(\tau ; t)=w^{i}\left(E_{\mathrm{N}}^{i}(\tau ; t), P_{\mathrm{N}}(\tau ; t)\right), g_{\mathrm{N}}^{i}(\tau ; t)=g^{i}\left(E_{\mathrm{N}}^{i}(\tau ; t)\right)$ and $l_{\mathrm{N}}^{i}(\tau ; t)=l^{i}\left(P_{\mathrm{N}}(\tau ; t)\right)$ the optimal instantaneous welfare, gains from emissions and losses from pollution of region $i$ at time $\tau \in[0, T]$ without cooperation.

\footnotetext{
7 And yet, this is not the only option; the new agreement could be renegotiated with a delay, or signed and broken many times within this period, or its length could be modified to a longer or shorter period, etc.
} 
Note that we are assuming existence and uniqueness for problems (2) and (3). Later, in Section 4, an application is presented with specific functional forms which guarantee the existence and uniqueness of the solutions.

2.2 The Imputation distribution procedure and main definitions

The main objective of this paper is to define an imputation distribution procedure, i.e. a dynamic sharing rule of the payoffs associated with current emissions under cooperation. Contrary to the standard literature, the proposed IDP does not rely on a particular solution concept, but it is defined in order to satisfy some desirable properties.

The distribution scheme is a flow of payoffs, $\pi^{i}(\tau)$, for any region $i \in\{1,2\}$ and at any time $\tau \in[0, T]$, which must first fulfill a feasibility condition at any time:

$$
\sum_{i=1}^{2} \pi^{i}(\tau)=\sum_{i=1}^{2} w_{\mathrm{C}}^{i}(\tau), \quad \forall \tau \in[0, T] .
$$

Condition (4) can alternatively be written as $\pi(\tau)=w_{\mathrm{C}}(\tau), \forall \tau \in[0, T]$. According to this condition, the sharing rule distributes the instantaneous payoff at each time between the two players. Instantaneous transfers can occur between the regions at every time $\tau \in[0, T]$, but payoffs can not be borrowed from or lent to the future. Define by $s d^{i}(\tau)$ the side-payment from region $-i$ to region $i$ under the IDP. It can be positive or negative, but from (4) always satisfies $s d^{i}(\tau)=-s d^{-i}(\tau)$, for any $\tau \in[0, T]$. We assume that only the instantaneous gains from production associated with emissions can be transferred between regions, the environmental losses, $l_{\mathrm{C}}^{i}(\tau)$, remain the same regardless of the form of the IDP. Then, the IDP for region $i$ can be written as:

$$
\pi^{i}(\tau)=g_{\pi}^{i}(\tau)-l_{\mathrm{C}}^{i}(\tau), \quad \text { with } g_{\pi}^{i}(\tau)=g_{\mathrm{C}}^{i}(\tau)+s d^{i}(\tau)
$$

Subscript $\pi$ denotes the cooperative payoff once the distribution procedure is implemented.

In addition to the feasibility condition (4), the IDP must fulfill three axioms: time consistency, a benefits pay principle, and a polluter pay principle. In order to define these axioms, one needs to characterize, for each player $i$ :

1. What he/she gets from a revision time $t \in[0, T]$ on.

The payoff to go that region $i$ gets from time $t$ onward if either cooperation is maintained from this time until $T$ under the proposed IDP $\pi^{i}(\tau)$, or conversely, if cooperation halts and both players play non-cooperatively therein can be written as:

$$
\begin{aligned}
& W_{\pi}^{i}(t)=\int_{t}^{T} \pi^{i}(\tau) e^{-\rho(\tau-t)} d \tau-D^{i}\left(P_{\mathrm{C}}(T)\right) e^{-\rho(T-t)} \\
& W_{\mathrm{N}}^{i}(t)=\int_{t}^{T} w_{\mathrm{N}}^{i}(\tau ; t) e^{-\rho(\tau-t)} d \tau-D^{i}\left(P_{\mathrm{N}}(T ; t)\right) e^{-\rho(T-t)}
\end{aligned}
$$


In the particular case when no redistribution scheme is implemented, each region gets its cooperative payoff without side-payments. Hence, the payoff to go for region $i$ at time $t$ would read:

$$
W_{\mathrm{C}}^{i}(t)=\int_{t}^{T} w_{\mathrm{C}}^{i}(\tau) e^{-\rho(\tau-t)} d \tau-D^{i}\left(P_{\mathrm{C}}(T)\right) e^{-\rho(T-t)} .
$$

2. His/her benefit from the cooperative agreement.

The gains from the agreement come in the form of a cleaner environment. To compute them, the cooperative solution must be compared against the non-cooperative solution assuming that cooperation has been maintained up until time $t \in(0, T)$, and the two regions play non-cooperatively from this moment on. The benefit from cooperation from time $t$ onward for region $i$ is defined as the reduction in the environmental damage from a less polluted environment, associated with lower emissions under cooperation, from $t$ to $T$ :

$$
B^{i}(t)=\int_{t}^{T}\left[l_{\mathrm{N}}^{i}(\tau ; t)-l_{\mathrm{C}}^{i}(\tau)\right] e^{-\rho(\tau-t)} d \tau+\left[D^{i}\left(P_{\mathrm{N}}(T ; t)\right)-D^{i}\left(P_{\mathrm{C}}(T)\right)\right] e^{-\rho(T-t)}, \quad t \in[0, T] .
$$

In the non-cooperative solution, optimal emissions in one region are computed by taking into account how one region's emissions increase the future pollution stock, and hence, the region's welfare. In the cooperative solution, the negative effect of pollution on the other region's welfare is also taken into account. In consequence, under cooperation the emissions and the pollution stock are kept lower at any time and, specifically, at the final time. Under cooperation, since both regions emit less, a lower pollution stock will imply positive gains from a cleaner environment: $B^{i}(t) \geq 0$ for all $i \in\{1,2\}$, $t \in[0, T]$.

3. His/her contribution to the cooperative agreement.

Contribution to the agreement by region $i$ comes in the form of losses from foregone emissions and production. They are computed comparing the non-cooperative gains from production with the gains under the proposed IDP:

$$
C_{\pi}^{i}(t)=\int_{t}^{T}\left[g_{\mathrm{N}}^{i}(\tau ; t)-g_{\pi}^{i}(\tau)\right] e^{-\rho(\tau-t)} d \tau, \quad t \in[0, T]
$$

Likewise, the contribution to the agreement if no distribution mechanism is implemented reads:

$$
C^{i}(t)=\int_{t}^{T}\left[g_{\mathrm{N}}^{i}(\tau ; t)-g_{\mathrm{C}}^{i}(\tau)\right] e^{-\rho(\tau-t)} d \tau, \quad t \in[0, T]
$$

Note that the benefits from cooperation in (8) are independent of the implemented redistribution scheme. Conversely, as observed above, the distribution scheme determines each regions' contribution $C_{\pi}^{i}(t)$. Nevertheless, the following proposition proves that the joint contribution is invariant to the chosen IDP.

Proposition 1 Defining the global contribution from time $t$ onward as $C_{\pi}(t)=\sum_{i=1}^{2} C_{\pi}^{i}(t)$ and $C(t)=\sum_{i=1}^{2} C^{i}(t)$. Then

$$
C_{\pi}(t)=C(t), \quad \forall t \in[0, T] \quad \text { and } \quad \forall \pi(t) \text { satisfying }(4)
$$


Proof Since $s d^{i}(\tau)=-s d^{i}(\tau)$ for any $\tau \in[0, T]$, then

$C_{\pi}(t)=\int_{t}^{T} \sum_{i=1}^{2}\left[g_{\mathrm{N}}(\tau ; t)-g_{\pi}(\tau ; t)\right] e^{-\rho(\tau-t)} d \tau=\int_{t}^{T} \sum_{i=1}^{2}\left[g_{\mathrm{N}}^{i}(\tau ; t)-g_{\mathrm{C}}^{i}(\tau ; t)\right] e^{-\rho(\tau-t)} d \tau=C(t)$.

Proposition 1 states that the joint contribution for the two players is the same for any IDP that satisfies Condition (4). In particular, it is equal to the joint contribution in the cooperative case prior to any redistribution scheme.

4. His/her responsibility from past emissions.

We define region $i$ 's net responsibility (or responsibility for shortness) as the damage that this region's accumulated past emissions have caused region $-i$, minus the damage that region $-i$ 's past emissions have caused region $i$. By past emissions we refer to emissions prior to the starting of cooperation. Therefore, responsibility is constant and independent on the actions taken while cooperating. This definition of responsibility takes into account the three major factors highlighted in Hayner and Weisbach (2016): who causes the problem and to what extent; what is the size of the harm caused; and to what extent each region has been impacted. Equivalently, responsibility can be defined as the total damaged caused by region $i$ which is not borne by this region. Define by $r^{i}$ all past emissions from region $i$ divided by all past emissions, i.e. the percentage of the initial pollution stock country $i$ is responsible for. Define the damage borne by region $i$ from all previous emissions as the damage associated with the initial pollution stock, $D^{i}\left(P_{0}\right)$. Then, this region's responsibility would read:

$$
R^{i}=r^{i} \hat{D}^{-i}\left(P_{0}\right)-r^{-i} \hat{D}^{i}\left(P_{0}\right), \quad \text { with } \quad \hat{D}^{i}\left(P_{0}\right)=\frac{D^{i}\left(P_{0}\right)}{\sum_{i=1}^{2} D^{i}\left(P_{0}\right)}
$$

Or alternatively,

$$
R^{i}=r^{i}-\widehat{D}^{i}\left(P_{0}\right)
$$

Expressions (12) and (13) are equivalent and it is immediately obvious that $R^{i}=-R^{-i}$. In general, the two regions are both responsible for past emissions although probably at different scales. We will say that region $i$ is responsible (or more responsible) if $R^{i}>0$ and not responsible (or less responsible if $\left.R^{i}<0\right)$. Moreover, responsibility does not change across time

Given the previous definitions of the payoffs to go, benefit, contribution and responsibility, we are now in the position to define the three desirable properties: individual rationality at any time $t$, a benefits pay principle and a polluter pay principle. These properties are defined in the following three axioms.

Axiom 1 (Time consistency) At any intermediate time $t$, and for each region $i$, the payoff to go under the distribution scheme $\pi$ is not lower than the payoff to go in the non-cooperative scenario:

$$
W_{\pi}^{i}(t) \geq W_{N}^{i}(t), \quad \forall t \in[0, T], \forall i \in\{1,2\}
$$


Axiom 2 (Benefits pay principle-BPP) All other things being equal, the greater one region's relative gain from cooperation the greater its relative contribution. Let a and b stand for two specific functional forms and sets of parameters values for gains and losses from pollution. Then, for any $t \geq 0$,

$$
\hat{B}_{a}^{i}(t)>\hat{B}_{b}^{i}(t) \Rightarrow\left(\hat{C}_{\pi}^{i}\right)_{a}(t)>\left(\hat{C}_{\pi}^{i}\right)_{b}(t)
$$

with $\hat{B}^{i}(t)=B^{i}(t) / B(t)$ and $\hat{C}_{\pi}^{i}(t)=C_{\pi}^{i}(t) / C(t)$ (a hat denotes the relative value of one region with respect to the total).

Moreover, we would like the distribution scheme to also take into account each region's responsibility for past emissions.

Axiom 3 (Responsibility or polluter's pay principal-PPP) All other things being equal, the greater one region's net responsibility for the damage caused by past emissions, ${ }^{8} R^{i}$, the greater its relative contribution. For any $t \geq 0$,

$$
\frac{\partial \hat{C}_{\pi}^{i}(t)}{\partial R^{i}}>0
$$

\section{The dynamic sharing rule}

A cooperative agreement is signed because it satisfies global rationality or Kaldor-Hicks efficiency. Globally, for the two regions, the payoff to go of maintaining cooperation surpasses the payoff to go in the non-cooperative scenario. The agreement is jointly profitable not only initially but at any intermediate time.

Remark 1 By virtue of joint maximization the global surplus to go linked to the cooperative solution is initially positive as well as at any ulterior time. That is, for all $t \in[0, T)$ :

$$
S(t)=\sum_{i=1}^{2}\left(W_{\mathrm{C}}^{i}(t)-W_{\mathrm{N}}^{i}(t)\right)=B(t)-C(t) \geq 0 .
$$

Besides global rationality, the distribution scheme must satisfy Condition (4), according to which the instantaneous joint payoff for the two regions under the IDP equates to the cooperative joint payoff. Thus, the IDP must determine how to share the instantaneous joint cooperative payoff at any time. Furthermore, this sharing rule must also guarantee axioms 1-3.

\subsection{A time-consistent IDP}

Time consistency implies that at every time $t$, each player prefers to follow the cooperative behavior rather than the non-cooperative one. Taking into account Remark 1, the statement in Axiom 1 can be re-defined in terms of the global surplus to go. An equivalent definition of time-consistency is proposed in next proposition.

\footnotetext{
8 Although we have presented a particular definition of net responsibility in Equations (12) or (13), alternative definitions are possible.
} 
Proposition 2 An IDP with the corresponding payoff to go $W_{\pi}^{i}(t)$ in (5), is time consistent and satisfies Condition (4) if and only if the following condition is satisfied at any time $t \in[0, T]$ :

$$
W_{\pi}^{i}(t)=W_{N}^{i}(t)+\phi^{i}(t) S(t) \quad \forall t \in[0, T]
$$

with $\phi^{i}(t)$ a differentiable function satisfying:

$$
\phi^{i}(t) \in[0,1], \quad \phi^{i}(t)+\phi^{-i}(t)=1, \quad \forall t \in[0, T], i \in\{1,2\}
$$

Proof See Appendix A.

This definition does not uniquely characterize a time-consistent IDP. A different IDP arises for each differentiable function $\phi^{i}(t)$ that satisfies Condition (17). Regardless of the chosen function $\phi^{i}(t)$, the joint payoff to go under the IDP equates to the cooperative payoff to go at any time $t: W_{\pi}(t)=$ $\sum_{i} W_{\pi}^{i}(t)=\sum_{i} W_{\mathrm{C}}^{i}(t)=W_{\mathrm{C}}(t), \forall t \in[0, T]$. Moreover, computing the derivative with respect to $t$ in both sides of this equation one finds that Condition (4) holds at every time.

Remark 2 An alternative way to write Condition (16) is

$$
\phi^{i}(t)=\frac{W_{\pi}^{i}(t)-W_{\mathrm{N}}^{i}(t)}{B(t)-C(t)}=\frac{B^{i}(t)-C_{\pi}^{i}(t)}{B(t)-C(t)}=\frac{S^{i}(t)}{S(t)}, \quad \forall t \in[0, T], \forall i \in\{1,2\} .
$$

Thus, $\phi^{i}(t)$ is the ratio of region $i$ 's surplus to go over the total surplus to go. Hence, it can be interpreted as a measure of the relative net gains for player $i$ if cooperation is maintained under a specific IDP from any time $t \in[0, T]$ onward.

According to the previous remark, once a differentiable function $\phi^{i}(t)$ that satisfies Conditions in (17) is chosen, then the net gains that each region obtains from the agreement are fully determined. Therefore, given the function $\phi^{i}(t)$ we can univocally characterize the flow of current payoffs under the IDP.

Remark 3 Let $\phi^{i}(t)$ be a differentiable function satisfying (17). Then, there is a unique time-consistent IDP which satisfies (16) and is characterized as:

$$
\pi^{i}(t)=w_{\mathrm{C}}^{i}(t)+\phi^{i}(t) I V C(t)-I V C^{i}(t)-\dot{\phi}^{i}(t) S(t)
$$

with $I V C^{i}(t)$, the instantaneous value of cooperation at time $t$, for region $i$ :

$$
\begin{aligned}
& I V C^{i}(t)=w_{\mathrm{C}}^{i}(t)-w_{\mathrm{N}}^{i}(t ; t)+ \\
& \int_{t}^{T} \dot{w}_{\mathrm{N}}^{i}(\tau ; t) e^{-\rho(\tau-t)} d \tau-\left(D^{i}\right)^{\prime}\left(P_{\mathrm{N}}(T ; t)\right) \dot{P}_{\mathrm{N}}(T ; t) e^{-\rho(T-t)} .
\end{aligned}
$$

and $I V C(t)=\sum_{i=1}^{2} I V C^{i}(t)$. This IDP satisfies Condition (4).

Proof See Appendix A. 
At time $t \in[0, T)$, if cooperation has been maintained until then, and if cooperation does not halt at this time, region $i$ would typically get a lower gains from production associated with lower emissions. This decrease in production, $g_{\mathrm{C}}^{i}(t)-g_{\mathrm{N}}^{i}(t ; t)<0$, defines the instantaneous effort to cooperate for region $i$ at time $t$. However, because cooperative regions emit less at time $t$, this would allow for higher future emissions for country $i$ and/or lower pollution stock than if the deviation from cooperation takes place at the instant immediately afterwards (the integral term in Expression (20)). Moreover, lower instantaneous emissions at time $t$ also induce a lower pollution stock from time $T$ on and therefore lower environmental damage borne by this region from $T$ onward (last term in expression (20)).

The IDP in (19) satisfies the feasibility Condition (4), which implies that the instantaneous payoff that the IDP distributes between the two players matches the total instantaneous cooperative payoff at every time $t$. Therefore, $\pi^{i}(t)-w_{\mathrm{C}}^{i}(t)$ defines the instantaneous side-payment to region $i$ (if positive), or from region $i$ (if negative).

According to the definition of the $I V C^{i}(t)$, the IDP in (19) grants the $i$-th player his instantaneous cooperative payoff, plus the gap between the share $\phi^{i}(t)$ of the joint instantaneous value of cooperation and the $i$-th player's instantaneous value of cooperation. Thus, if the $i$-th IVC is larger than its share of the total IVC, this region would transfer part of its instantaneous payoff to its opponent. Furthermore, this instantaneous side-payment is increased at the speed in which his share of the total surplus to go increases.

Remark 4 While Proposition 2 characterizes a time-consistent IDP, it is also applicable to characterize agreeability. ${ }^{9}$ This latter is a stronger concept of dynamic individual rationality. For a time-consistent sharing mechanism, conditions (16) and (17) must be satisfied under the assumption that cooperation has been followed from time 0 until time $t$. An agreeable IDP requires that these conditions are satisfied regardless of the path $P(t)$ followed from 0 to $t$, i.e. at any position of the game.

Agreeability is a more general concept, which guarantees the stability of the cooperative agreement, even if one of the players deviates from cooperation for a given period of time, which is more likely to occur the larger the number of agents involved. Agreeability is also an adequate sustainability concept when exogenous shocks that shift the pollution stock out of the initially optimal cooperative path are likely.

We focus on time consistency because we are considering only two players and because we have assumed that once cooperation halts, players move to their non-cooperative mode of play thereafter.

Any differentiable function $\phi^{i}(t)$, which satisfies the conditions in (17), guarantees time-consistency (and agreeability). Among these functions, we look for the specification(s) which also implies the satisfaction of axioms 2 and 3 .

\footnotetext{
9 Agreeability was first introduced by Kaitala and Pohjola (1990). The connection between time consistency and agreeability are studied in Jørgensen et al. (2003, 2005).
} 
Given the definition of responsibility in (12) and (13), the next proposition defines $\phi^{i}(t)$, as a function of $\hat{B}^{i}(t)$ and $R^{i}$, for which the IDP $\pi$ fulfills the desired axioms 2 and 3 .

Proposition 3 Let $\alpha(t) \geq 0$ be given, then for any $t \in[0, T]$ define $\phi^{i}(t)$ as: ${ }^{10}$

$$
\phi^{i}(t)= \begin{cases}0 & \text { if } R^{i}>0 \wedge \alpha(t) \geq \alpha_{\max }(t), \\ \hat{B}^{i}(t)-\alpha(t) \frac{C(t)}{S(t)} R^{i} & \text { if } \alpha(t) \in\left[0, \alpha_{\max }(t)\right) . \\ 1 & \text { if } R^{i}<0 \wedge \alpha(t) \geq \alpha_{\max }(t),\end{cases}
$$

with

$$
\alpha_{\max }(t)=\frac{\hat{B}^{j}(t)}{R^{j}} \frac{S(t)}{C(t)}, \quad j=\underset{i \in\{1,2\}}{\arg \max }\left\{R^{i}\right\} .
$$

Then, the relative contribution of region $i$ reads:

$$
\hat{C}_{\pi}^{i}(t)= \begin{cases}\hat{B}^{i}(t) \frac{B(t)}{C(t)} & \text { if } R^{i}>0 \wedge \alpha(t) \geq \alpha_{\max }(t), \\ \hat{B}^{i}(t)+\alpha(t) R^{i} & \text { if } \alpha(t) \in\left[0, \alpha_{\max }(t)\right) \\ \hat{B}^{i}(t) \frac{B(t)}{C(t)}-\frac{S(t)}{C(t)} & \text { if } R^{i}<0 \wedge \alpha(t) \geq \alpha_{\max }(t) .\end{cases}
$$

And the IDP, $\pi$, defined in (19), satisfies axioms 2 and 3 whenever $\alpha(t) \in\left(0, \alpha_{\max }(t)\right)$.

Proof The relative contribution in (23) comes from (18) and (21). The interior expression for the relative contribution straightforwardly satisfies axioms 2 and 3.

According to (21), the relative net gains of region $i$ are greater the higher this region's relative benefit from the cooperative agreement, $\hat{B}^{i}(t)$. Furthermore, $\phi^{i}(t)$ shrinks (resp. widens) with region $i$ 's net responsibility, if positive (resp. negative). In this definition, the responsibility is valued by the effort or contribution which is required to achieve a unit of surplus. The weight given to responsibility with respect to the relative benefit is measured by $\alpha(t)$. If this weight is too large, $\alpha(t)>\alpha_{\max }(t)$, then the region which is more responsible for past emissions gets no share of the total surplus to go, which is fully assigned to the region less responsible. However, if the weight given to responsibility does not fully offset the importance of the relative benefit, then both agents get a positive share of the surplus to go from time $t$ (i.e., an interior expression with $\left.\phi^{i}(t) \in(0,1)\right)$. The weight $\alpha(t)$ can be assumed constant, or it can depend on time (for example, a lower weight to responsibility as the agreement advances).

In the interior case, when $\alpha(t) \in\left[0, \alpha_{\max }(t)\right)$, the relative contribution of region $i$ in $(23)$ is determined by its relative gains from cooperation increased by a fraction $\alpha(t)$ of its net responsibility for past emissions (decreased if the region has a negative net responsibility). Thus, in the interior case, the

\footnotetext{
10 In fact, the interior expression applies for $\alpha(t) \in\left[0, \alpha_{\max }(t)\right)$, whatever the value of $R^{i}$; but also for $R^{i}=0$ whatever the value of $\alpha(t)$. We do not write down this latter case for brevity. This particular case will be commented on the following subsection.
} 
relative contribution is positively correlated with the relative benefit and with the responsibility, and therefore it fulfills Axiom 2 as well as Axiom 3.

If the weight given to responsibility is very large, $\alpha(t)>\alpha_{\max }(t)$ and region $i$ is responsible, then its relative contribution is given by a ratio defined by its relative benefit, scaled up by the benefit per unit of contribution, $B(t) / C(t)>1$. Conversely, if region $i$ is not responsible, then this ratio is reduced in the total surplus to go per unit of contribution.

The contribution of region $i$ in absolute terms, reads:

$$
C_{\pi}^{i}(t)= \begin{cases}B^{i}(t) & \text { if } R^{i}>0 \wedge \alpha(t) \geq \alpha_{\max }(t), \\ B^{i}(t) \frac{C(t)}{B(t)}+\alpha(t) R^{i} C(t) & \text { if } \alpha(t) \in\left[0, \alpha_{\max }(t)\right) \\ B^{i}(t)-S(t) & \text { if } R^{i}<0 \wedge \alpha(t) \geq \alpha_{\max }(t) .\end{cases}
$$

This expression shows that, for the interior case, at time $t$, each region contributes an equal share, $C(t) / B(t)$ of its benefit from cooperation. Furthermore, if the region is responsible for past emissions, its contribution rises by a share $\alpha(t) R^{i}$ of the total contribution (which is subtracted to the nonresponsible region). If the weight given to responsibility is too large, $\alpha(t)>\alpha_{\max }(t)$, and if region $i$ is responsible, the contribution of this region from any given time $t$ onward equals its benefits from cooperation from this moment onward. This is the maximum amount that this region can contribute compatible with time consistency. Conversely, if the region is not responsible, then its contribution is given by its benefits decreased by the total surplus from this time onward.

To summarize, the IDP $\pi^{i}(t)$ defined in (19) and (20), with the proposed specification for $\phi^{i}(t)$ in $(21)$ is time consistent. Furthermore, whenever $\alpha(t) \in\left(0, \alpha_{\max }(t)\right)$, it also satisfies the benefits pay principle and the polluter pay principle.

In the particular case without asymmetric responsibility, or if the responsibility principle was ignored, the following remark can be obtained.

Remark 5 From (23) it is straightforward to conclude that, if $\alpha=0$, or if $R^{i}=R^{-i}=0$ (i.e. $r^{i}=\hat{D}^{i}\left(P_{0}\right)$ ), then:

$$
\hat{C}_{\pi}^{i}(t)=\hat{B}^{i}(t), \quad C_{\pi}^{i}(t)=B^{i}(t) \frac{C(t)}{B(t)}
$$

If all the weight is given to the benefits pay principle $(\alpha(t)=0$ for all $t \geq 0)$, or both regions are equally responsible $\left(R^{i}=0\right)$, then the relative contribution matches the relative benefit from cooperation for both regions. Each region pays the same percentage of its benefits from cooperation. A distribution procedure which strives for an exact equivalence between relative gains and relative contributions would be uniquely characterized by (19) with function $\phi^{i}(t)=\hat{B}^{i}(t)$, that is, disregarding the PPP or responsibility axiom, $\alpha(t)=0$. 
This subsection explores the links between our proposal and the Nash bargaining solution under symmetric and asymmetric specifications.

Proposition 4 For the proposed IDP in (19), (20) and (21), if the region who is net responsible for past emissions benefits the least from the agreement, then it never holds true that $\phi^{i}(t)=1 / 2$. The egalitarian rule never arises.

Proof If region $i$ is net responsible and benefits the least from the agreement $\left(R^{i}>0\right.$ and $\left.\hat{B}^{i}(t)<1 / 2\right)$, then from $(21)$ it is immediately obvious that $\phi^{i}(t) \in[0,1 / 2)$ for any $\alpha \geq 0$. Likewise, if region $i$ is not responsible and benefits the most from the agreement $\left(R^{i}<0\right.$ and $\left.\hat{B}^{i}(t)>1 / 2\right)$, then $\phi^{i}(t) \in(1 / 2,1]$ for any $\alpha \geq 0$.

Proposition 4 rules out the egalitarian rule or the symmetric Nash bargaining solution. Interestingly, the payoffs to go from our proposed IDP, when players agree to share the surplus to go according to the expression $\phi^{i}(t)$ in (21), would equivalently arise from the asymmetric Nash bargaining solution in which $\phi^{i}(t)$ defines region $i$ 's bargaining power.

Proposition 5 The payoffs to go at time $t, W_{\pi}^{i}(t), i \in\{1,2\}$, which satisfy Condition (16) are equivalent to the payoffs stemming from an asymmetric Nash bargaining solution of the form:

$$
\begin{aligned}
& \max _{W_{\pi}^{i}(t), i \in\{1,2\}} \prod_{i=1}^{2}\left(W_{\pi}^{i}(t)-W_{N}^{i}(t)\right)^{\phi^{i}(t)} \\
& \text { s.t.: } \sum_{i=1}^{2} W_{\pi}^{i}(t)=W_{C}(t) \quad \text { and } \quad(17) .
\end{aligned}
$$

Proof Let us remove the time argument. Problem (25)-(26) can be rewritten as:

$$
\max _{W_{\pi}^{1}}\left(W_{\pi}^{1}-W_{\mathrm{N}}^{1}\right)^{\phi^{1}}\left(W_{\mathrm{C}}-W_{\pi}^{1}-W_{\mathrm{N}}^{2}\right)^{1-\phi^{1}}
$$

Taking the derivative wrt $W_{\pi}^{1}$, and if the non-cooperative solution $\left(W_{\pi}^{i}=W_{\mathrm{N}}^{i}\right)$ is discarded, the FOC for this problem reads:

$$
\left(\frac{W_{\mathrm{C}}-W_{\pi}^{1}-W_{\mathrm{N}}^{2}}{W_{\pi}^{1}-W_{\mathrm{N}}^{1}}\right)^{1-\phi^{1}}\left\{\phi^{1}-\left(1-\phi^{1}\right) \frac{W_{\pi}^{1}-W_{\mathrm{N}}^{1}}{W_{\mathrm{C}}-W_{\pi}^{1}-W_{\mathrm{N}}^{2}}\right\}=0 .
$$

And by equating the second term in brackets to zero, it immediately follows that $W_{\pi}^{1}=W_{\mathrm{N}}^{1}+\phi^{1} S(t)$. And therefore $W_{\pi}^{2}=W_{\mathrm{N}}^{2}+\phi^{2} S(t)$.

Proposition 5 establishes the equivalence between the bargaining power of region $i$ and the share of the total surplus assigned to this region by the proposed IDP. Therefore, from equation (21) there is a one-to-one relationship between the bargaining power of the two regions and the weight given to responsibility in the proposed IDP. This relationship can be interpreted in two opposite direction. On the one hand, assume that the bargaining power of the two regions are known at a given time $t$. Then, 
these values determine what weight should be assigned to responsibility in the proposed IDP. From (21), this weight reads:

$$
\alpha(t)=\frac{\hat{B}^{i}(t)-\phi^{i}(t)}{C(t) R^{i}} S(t) .
$$

Interestingly, assuming that $i$ is the responsible region (i.e. $R^{i}>0$ ), then a condition for a positive valuation of responsibility emerges:

$$
R^{i}>0 \Rightarrow\left(\alpha(t)>0 \Leftrightarrow \phi^{i}(t)<\hat{B}^{i}(t)\right)
$$

Thus, players' bargaining power would be compatible with a sharing rule assigning positive weight to responsibility only if the region with positive responsibility has a bargaining power below its relative benefit from the agreement. Otherwise, if $\phi^{i}(t) \geq \hat{B}^{i}(t)$ for the responsible region, then responsibility would play no role in the IDP. The responsible region would succeed in ruling out the responsibility or pollution pay principle.

On the other hand, if the bargaining power is not given, then a specific value/function $\alpha(t)$ needs to be determined. Either the parties agree on a particular specification, or $\alpha(t)$ is chosen according to some desirable property. For example, one possibility is to choose $\alpha(t) \in\left[0, \alpha_{\max }(t)\right]$ in order to minimize transfers, assuming transaction costs. Given this weight, the share of the total surplus assigned to each region by the proposed IDP can be computed by (21).

In the next section we follow this second interpretation and, for a given $\alpha(t)$ (indeed constant), the corresponding $\phi^{i}(t)$ and the proposed IDP are computed. The dynamic sharing mechanism is applied to a particular example, considering particular functional forms and parameters values to illustrate the main findings of the paper.

\section{Application of the IDP to a particular example}

In this section, we describe how the IDP presented in the previous section can be applied to a specific example. Functions are chosen to ease computation maintaining, at the same time, the essence of the asymmetric environmental problem.

1. The pollution stock dynamics in (1) is described as a linear differential equation:

$$
F\left(E^{1}(\tau), E^{2}(\tau), P(\tau)\right)=E^{1}(\tau)+E^{2}(\tau)-\delta P(\tau)
$$

with $\delta$ the degree of assimilative capacity of the environment.

2. The effect of pollution during cooperation is negligible. We are interested in environmental problems characterized by a current sacrifice, in terms of emissions reduction throughout the cooperative period, overcompensated by lower environmental losses, fundamentally in the future. Thus, for 
simplicity, we assume that pollution has no damaging effect throughout the cooperative period and it does not affect production either: ${ }^{11} w^{i}\left(E^{i}(\tau), P(\tau)\right)=g^{i}\left(E^{i}(\tau)\right), \forall \tau \in[0, T]$.

3. The damage is multiplicatively separable in a region-specific parameter, $d^{i}$, and a function of pollution, $D(P)$, identical for the two regions: $D^{i}(P)=d^{i} D(P)$. Several facts are true under this specification:

i) The relative damage from pollution for region $i$ does not depend on the level reached by the stock of pollution at $T$. Likewise, the relative gains obtained by this region (if it remains in the agreement from $t$ until $T$ ) is independent of the time $t$.

$$
\hat{D}^{i}(P)=\frac{d^{i}}{\sum_{j=1}^{2} d^{j}} ; \quad \hat{B}^{i}(t)=\frac{D^{i}\left(P_{\mathrm{N}}(T ; t)\right)-D^{i}\left(P_{\mathrm{C}}(T)\right)}{\sum_{j=1}^{2}\left(D^{j}\left(P_{\mathrm{N}}(T ; t)\right)-D^{j}\left(P_{\mathrm{C}}(T)\right)\right)}=\frac{d^{i}}{\sum_{j=1}^{2} d^{j}} .
$$

In fact, the two time-independent expressions coincide, $\hat{B}^{i}(t)=\hat{D}^{i}(P)=\hat{D}^{i}=d^{i} / \sum_{j=1}^{2} d^{j}$.

ii) The net responsibility of region $i$ reads:

$$
R^{i}=r^{i}-\hat{D}^{i}\left(P_{0}\right)=r^{i}-\hat{B}^{i}(t)
$$

Interestingly, in this case the responsibility shows a one-to-one negative relation with the relative benefit from the agreement.

iii) From (22) the upper bound for $\alpha$ in order to have an interior relative contribution in (23) is:

$$
\alpha_{\max }(t)=\frac{\hat{B}^{j}}{R^{j}} \frac{S(t)}{C(t)}=\frac{d^{j}}{r^{j} d^{-j}-r^{-j} d^{j}} \frac{S(t)}{C(t)}, \quad j=\underset{i \in\{1,2\}}{\arg \max }\left\{R^{i}\right\} .
$$

iv) From (21), the expression $\phi^{i}(t)$ easily follows under this specification of the damage function. For the interior case, $\alpha \in\left[0, \alpha_{\max }(t)\right)$ :

$$
\phi^{i}(t)=\hat{B}^{i}\left(1+\alpha \frac{C(t)}{S(t)}\right)-\alpha \frac{C(t)}{S(t)} r^{i}
$$

Although the relative damage and the relative benefit are time independent, the contribution required to attain one unit of surplus, $C(t) / S(t)$, is not constant, and neither is $\phi^{i}(t)$. If the contribution per unit of surplus increases across time, then the region which is net responsible will see its relative net benefit reduced across time to the benefit of the region which in not responsible). ${ }^{12}$ Opposite reasoning would apply if the contribution per unit of surplus decreases across time.

v) The relative contribution is constant in the interior case, where $\alpha \in\left[0, \alpha_{\max }(t)\right)$.

$$
\hat{C}_{\pi}^{i}(t)=\hat{C}_{\pi}^{i}=r^{i}+(1-\alpha) \hat{B}^{i}, \quad \forall t \in(0, T)
$$

More interestingly, the relative benefit for region $i$ has a twofold effect on this region's relative contribution. A higher $\hat{B}^{i}$ directly induces a larger relative contribution, $\hat{C}_{\pi}^{i}$, as stated by the

\footnotetext{
11 Since we are dealing with a stock pollution problem, the accumulated emissions within the cooperative period will have lasting effects. We focus on these effects, which can be specially important in the case of non-linear effects on ecosystems or irreversibilities.

$12 \phi^{i}(t)$ is related to $C(t) / S(t)$ negatively if $r^{i}>\hat{B}^{i}$ ( $i$ is responsible); and positively if $r^{i}<\hat{B}^{i}$ ( $i$ is not responsible).
} 
BPP. However, under a multiplicatively separable damage function, from (27) it also implies lower responsibility, which due to the PPP calls for a lower relative contribution. This defines an indirect negative effect of relative benefits on relative contributions. The direct positive effect outweighs the indirect negative effect if $\alpha<1$. Under this condition, a strong version of Axiom 2 (strong-BPP) is satisfied:

$$
\frac{d \hat{C}_{\pi}^{i}(t)}{d \hat{B}^{i}}>0, \quad \forall \alpha \in\left[0, \min \left(\alpha_{\max }(t), 1\right)\right) .
$$

4. For tractability, the instantaneous gains from production linked to current emissions are described by a linear quadratic function. Likewise, the damage from the pollution stock at time $T$ is assumed to be quadratic, and hence, multiplicatively separable,

$$
g^{i}\left(E^{i}(\tau)\right)=a^{i} E^{i}(\tau)-\frac{\left(E^{i}\right)^{2}(\tau)}{2}, \quad D^{i}(P(T))=d^{i} P^{2}(T) .
$$

Consider two regions who share a polluted environment. Region 1's responsibility exceeds its share of the burden from current pollution and hence $R^{1}=-R^{2}>0$. Moreover, Region 2 is more severely hit by the environmental problem, or equivalently, it will bear more damage if no agreement on emissions reduction is implemented, $\hat{B}^{2}(t)>\hat{B}^{1}(t)$.

Under these assumptions, we compute the cooperative and the non-cooperative solutions, see a sketch on how to compute them in Appendix B. Because we cannot obtain an analytical solution for the non-cooperative case, we rely on numerical simulations considering the following parameters values:

$$
r^{1}=0.72,\left(r^{2}=0.28\right), d^{1}=0.1<d^{2}=0.12, a=1, \delta=0.1, P_{0}=1, \rho=0.03
$$

We make the assumption that $a^{2}=a^{1}=a$ to clearly state that the two countries only differ in their responsibility for past emissions and their valuation of a cleaner environment. Thus, the marginal gains for additional emissions (or the cost of abatement) are identical in both regions. ${ }^{13}$ Moreover, $d^{2}>d^{1}>0$, implying that region 2 will be more strongly hit by the environmental problem. At the same time, we consider that the ratio of past emissions is relatively larger for Region 1.

Note first that these parameters are compatible with the assumption that Region 1 is responsible, while it benefits the least from the agreement. Because the damage function in (32) is multiplicatively separable, region $i$ 's relative benefit from cooperation is constant across time, and indeed is equal to the relative damage at the beginning of the agreement: $\hat{B}^{i}(t)=\hat{B}^{i}=\hat{D}^{i}(P)=d^{i} / \sum_{j=1}^{2} d^{j}$, with $\hat{B}^{1}=0 . \widehat{45}<\hat{B}^{2}=0.54$. Region 1 has a positive net responsibility, $R^{1}=0.1655>0$, while conversely, for Region $2, R^{2}=-R^{1}<0$.

Our numerical illustration highlights that, depending on the parameters values, the cooperative agreement either is already time consistent, or the IDP guarantees time consistency. For the parameters

13 This assumption can be easily removed, introducing asymmetry in production technologies, $a^{1} \neq a^{2}$. All the parameters values in (33) are chosen for illustration purposes. 
in (33), as Figure 1 (left) shows, the payoffs to go under cooperation surpass the non-cooperative payoffs to go for each region and at any time $t \in[0, T]$ and cooperation is time consistent. However, for different parameters, for example, rising $d_{2}$ up to 0.15 , Region 1 would deviate from cooperation unless an appropriate IDP is defined, as shown in Figure 1 (right). ${ }^{14}$
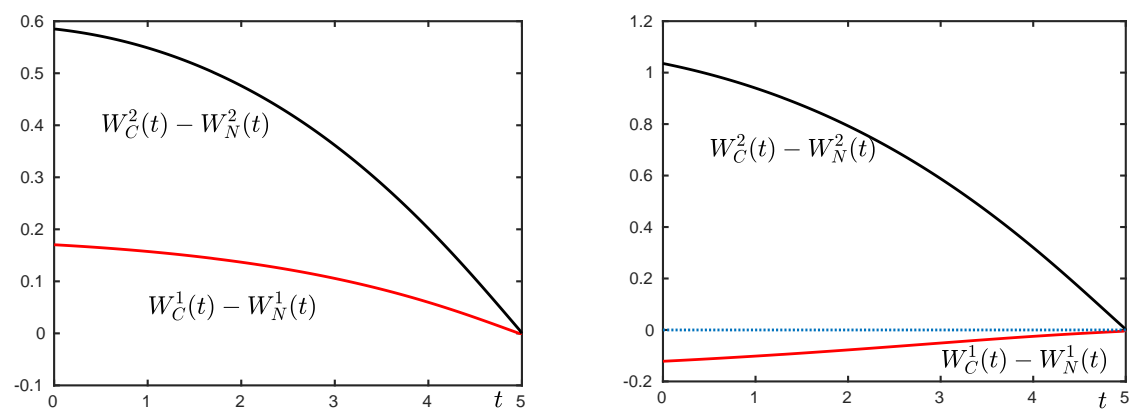

Fig. $1 W_{\mathrm{C}}^{i}(t)-W_{\mathrm{N}}^{i}(t)$ for $d_{2}=0.12$ (left) and $d_{2}=0.15$ (right).

Next we analyze the effect of the weight given to responsibility, $\alpha$, which is assumed constant across the cooperative period. It influences each region's share of the surplus to go, each region's contribution, and each region's payoff under the proposed redistribution scheme. We present the results for different values of $\alpha$ running from 0 to 1 . As shown in Expression (23) the responsibility Axiom 3 applies for $\alpha>0$. Likewise, $\alpha<1$ is a necessary condition for the strong-BPP defined in (31).

First, notice that in this example the contribution per unit of surplus, $C(t) / S(t)$, increases across time; or equivalently, the benefits per unit of contribution, $B(t) / C(t)$, decreases across time. ${ }^{15}$ As this ratio increases, the share of the total surplus to go allocated to the more-responsible region, $\phi^{1}(t)$, shrinks; while the share allocated to the less-responsible region, $\phi^{2}(t)$, enlarges. This is shown in Figure 2 (right) which depicts the evolution of $\phi^{i}(t, \alpha)$ as time runs from 0 to 5 for different values of $\alpha$ (in this illustrative section we have added an $\alpha$ argument to those functions which depend on this parameter).
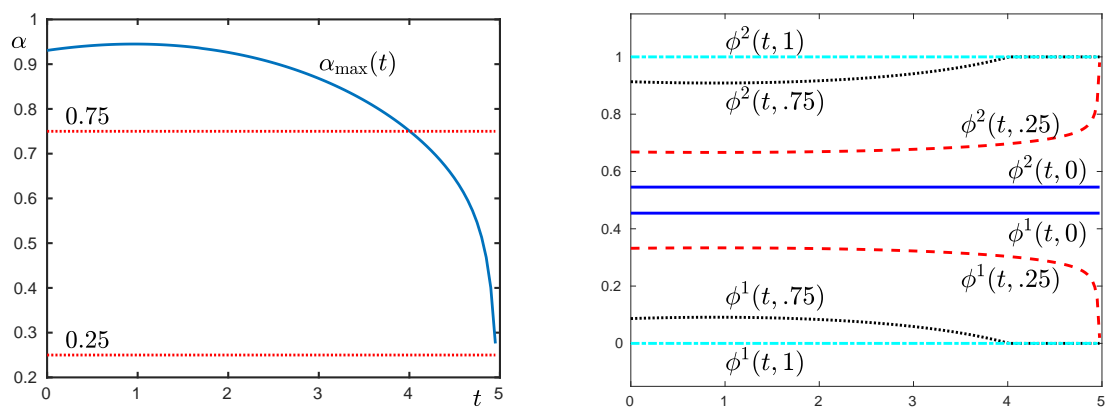

Fig. $2 \alpha_{\max }(t)$ (left); and $\phi(t, \alpha)$ for $\alpha=0,0.25,0.75,1$ (right).

\footnotetext{
14 We maintain parameters in (33) to better illustrate the effect of $\alpha$ on our results, which is one of the main interests of this section.

15 The inverse relation between $C(t) / S(t)$ and $B(t) / C(t)$ becomes immediately clear since $S(t)=B(t)-C(t)$.
} 
Notice that for (28), and since $C(t) / S(t)$ increases across time, then $\alpha_{\max }(t)$ is a decreasing function of time as shown in Figure 2 (left). Therefore, at the end of the planning horizon, solution may cease to be interior.

If no weight is given to responsibility, $\alpha=0, \phi^{i}(t)$ equals the constant $\hat{B}^{i} \in\{0 . \widehat{45}, 0.5 \overline{4}\}$. If little weight is given to responsibility, $\alpha=0.25, \phi^{i}(t)$ is the interior solution in (21) at any time and therefore, $\phi^{1}(t)$ decreases and $\phi^{2}(t)$ increases across time as $C(t) / S(t)$ falls down. If $\alpha=0.75$ it surpasses $\alpha_{\max }(t)$ before the end of the cooperative period (about $t=4)$. At this time $\phi^{1}(t)$ and $\phi^{2}(t)$ become 0 and 1 respectively, and remain at these values henceforth. In this last sub-interval, the entire surplus to go from cooperation goes to Region 2, and hence, Region 1 is indifferent towards cooperating or defecting. Finally, if $\alpha=1$ it surpasses $\alpha_{\max }(t)$ from the very beginning and $\phi^{1}(t, 1)=0$ at any time $t \in[0,5]$. The responsibility principle is so strong that the IDP allocates all the surplus to the less-responsible Region 2 right from the beginning of cooperation.

As shown in Figure 2 (right), the greater the weight given to responsibility, $\alpha$, the lower the share of the total surplus to go for the more-responsible Region 1 and the greater the share to the less-responsible Region 2. For Region 1, since $R^{1}>0$ then $\phi^{1}(t, \alpha) \leq \phi^{1}(t, 0)=\hat{D}^{1}=0 . \widehat{45}<1 / 2$. Similarly, for Region 2 , $R^{2}<0$ and then $\phi^{2}(t, \alpha) \geq \phi^{2}(t, 0)=\hat{D}^{2}=0.54>1 / 2$. Thus, regardless of the value of $\alpha$, Region 1 gets less than $1 / 2$ of the surplus and Region 2 gets more than $1 / 2$ of the surplus, as stated in Proposition 4 .

The effect of a greater weight given to responsibility can be equally observed in each region's relative contribution, $\hat{C}_{\pi}^{i}(t, \alpha)$. As $\alpha$ increases, the relative contribution of the most-responsible region also rises, while the relative contribution of the least-responsible region decays (see Figure 3 ). The relative contribution is constant across time in the interior, $\alpha \in\left[0, \alpha_{\max }(t)\right)$. However, for $\alpha \geq \alpha_{\max }(t)$ the contribution of the most-responsible Region 1 is defined by a share $B(t) / C(t)$ of its relative benefit $\hat{B}^{1}$. Because, in the example, the benefit per unit of contribution decreases across time, so too does the relative contribution of Region 1 . And correspondingly, the relative contribution of the least-responsible Region 2 increases.
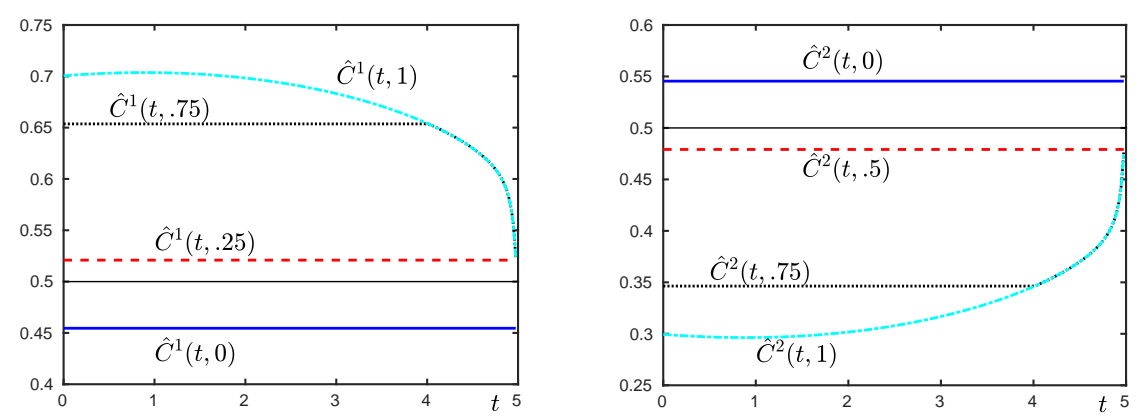

Fig. $3 \hat{C}_{\pi}^{i}(t, \alpha)$ for $\alpha=0,0.25,0.75,1$.

Figure 4 compares cooperative and non-cooperative payoffs before and after the implementation of the IDP. It depicts the payoffs to go from any given time $t$ onward, in the non cooperative case, $W_{\mathrm{N}}^{i}(t)$, 
and in the cooperative case without a redistribution scheme, $W_{\mathrm{C}}^{i}(t)$, or once the IDP is implemented, $W_{\pi}^{i}(t, \alpha)$ (in this latter case we distinguish two extremes: $\alpha=0$ and $\alpha=1$ ). The cooperative payoff to go exceeds the non-cooperative payoff to go for each region and at any time, $W_{\mathrm{C}}^{i}(t)>W_{\mathrm{N}}^{i}(t)$, for any $i \in\{1,2\}$ and any $t \in[0, T)$. Moreover, if the IDP is implemented disregarding responsibility, $\alpha=0$, the payoff increases for the responsible region and decreases for the non-responsible region. Conversely, if we move to the other extreme compatible with the strong-BPP in this example, $\alpha=1$, the responsible region sees its payoff reduced and the non-responsible region gets a higher payoff to go than before the implementation of the IDP. At any time $t$, the gap $W_{\pi}^{i}(t, \alpha)-W_{\mathrm{C}}^{i}(t, \alpha)$ defines the side-payment to go (the total side-payment from this time onward) that region $i$ would get from region $-i$. If negative, the side-payment would conversely flow from $i$ to $-i$. Thus a side-payment flows towards the more responsible region for small $\alpha$ and vice versa. Finally, Figure 4 clearly states that, for any intermediate $\alpha$ between 0 and 1, the IDP makes the agreement time consistent.
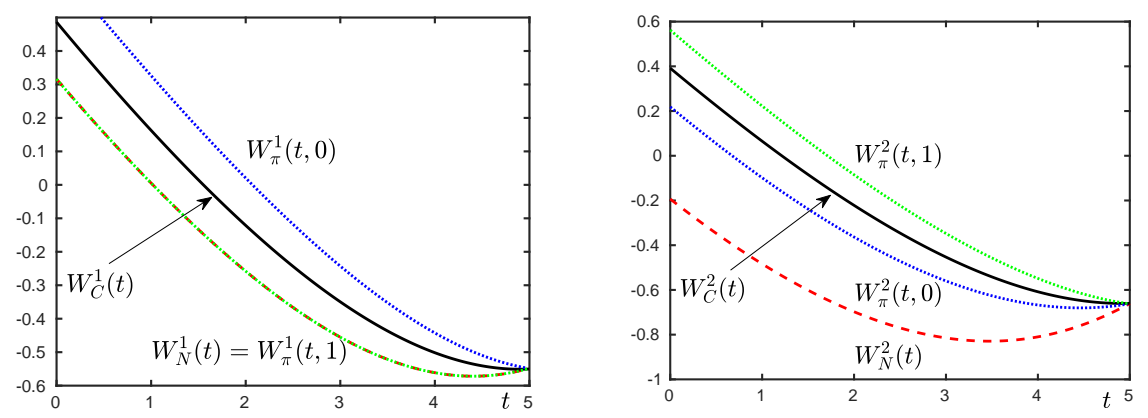

Fig. $4 W_{\mathrm{C}}^{i}(t), W_{\mathrm{N}}^{i}(t), W_{\pi}^{i}(t, \alpha)$ for $\alpha=0,1$.

Figure 4 encompasses ongoing gains from emissions and damage from pollution. A similar analysis can be made to compare the instantaneous payoffs at a specific instant of time $t$ in Figure $5 .{ }^{16}$ The instantaneous payoff is the greatest with no cooperation (the red-dashed line), because the two regions make no effort to reduce emissions. Cooperation comes with the associated cost of lower emissions across the whole cooperative period, which defines each region's contribution. The instantaneous cooperative payoff, without any side-payment, is depicted by the solid black line. An IDP that disregards responsibility, $\alpha=0$, would imply a lower effort for Region 1 and a higher effort for Region 2, than a cooperative agreement without any redistribution scheme. At each time $t$, when $\alpha=0$, the gap $\pi^{1}(t, 0)-w_{\mathrm{C}}^{1}(t)$ defines an instantaneous payoff transfers from Region 2 to Region 1 . By contrast, when the responsibility principle is strong $\alpha=1$, then the situation is reversed. The instantaneous transfer $\pi^{2}(t, 1)-w_{\mathrm{C}}^{2}(t)$ flows from Region 1 to Region 2 . The former increases its contribution and the latter reduces its contribution.

\footnotetext{
16 The non-cooperative instantaneous payoffs, $w_{\mathrm{N}}^{i}(t)$ (in Figure 5), are the highest across the cooperative agreement. However the non-cooperative payoffs to go, $W_{\mathrm{N}}^{i}(t)$ (in Figure 4), are the lowest. This reflects the strong effect of the pollution damage which only occurs once cooperation has halted and it is not accounted for by the instantaneous payoffs.
} 

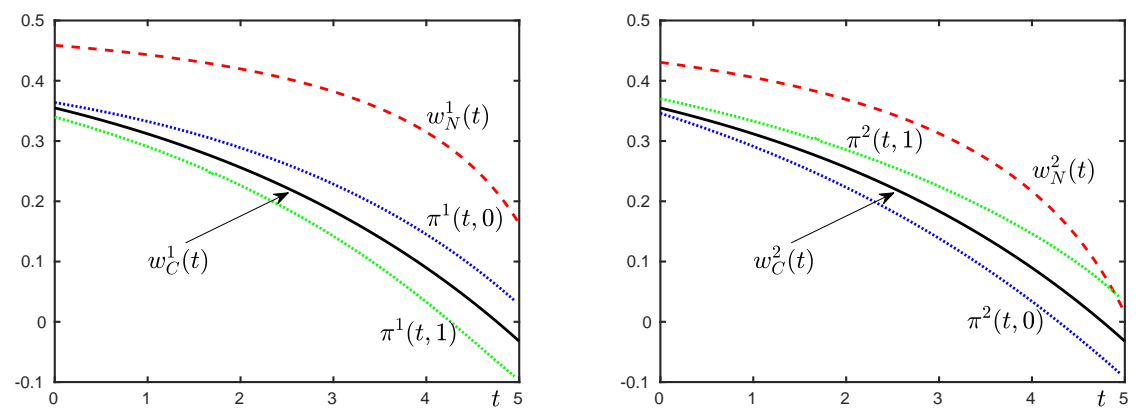

Fig. $5 w_{\mathrm{C}}^{i}(t), w_{\mathrm{N}}^{i}(t), \pi^{i}(t, 0), \pi^{i}(t, 1)$.

Finally, in Figure 6, we illustrate the strong-BPP defined in Expression (31). For $\alpha=0.75$ we compute the relative contribution of Region 1 for $d^{1}=0.1$ and for $d^{1}=0.11$. A rise in $d^{1}$ implies an increment in Region 1's relative benefit from $\hat{B}^{1}=0 . \widehat{45}$ to 0.4783 . This implies a reduction in this region's responsibility, from $R^{1}=0.2655$ to 0.2417 . A higher $d^{1}$ also increases $\alpha_{\max }(t)$ as displayed in Figure 6 (left) by the upward shifts from the blue-solid line to the red-dashed line. Whenever $\alpha$ remains below $\alpha_{\max }(t)$ for $d^{1}=0.1$ and $d^{1}=0.11$ (the relative contribution is given in $(30)$ ), the total effect of a rise in $d^{1}$ is a constant increment ${ }^{17}$ of the relative contribution of Region 1 from 0.6536 to 0.6596 . In the example, we observe that the strong-BPP holds true not only for the interior case, but also after the instant at which $\alpha$ surpasses $\alpha_{\max }(t)$ and the solution is no longer at the interior $\left(\phi^{1}(t)=0\right.$ and $\left.\phi^{2}(t)=1\right)$.
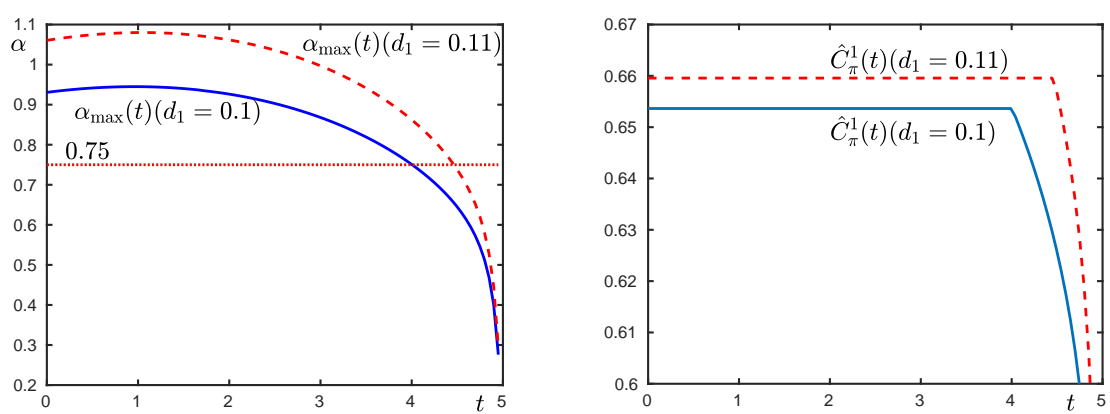

Fig. $6 \alpha_{\max }(t)$ for $d_{1}=0.1,0.11$ (left); and $\hat{C}_{\pi}^{i}(t, 0.75)$ for $d_{1}=0.1,0.11$ (right).

\section{Length of the cooperative agreement}

The main idea of our paper has been to characterize a sharing mechanism that together with time consistency satisfies two axioms: benefits pay principle and responsibility. Responsibility is defined taking into account past emissions (prior to the agreement). Because the cooperative agreement is finite, one can argue that the emissions within this period are relatively small with respect to all past emissions. Therefore, we believe that it is reasonable to demand the country which is responsible at the beginning of the agreement to bear a higher cost in terms of emissions reductions, ceteris paribus.

17 This increment corresponds to $(1-\alpha) \Delta \hat{B}^{1}=0.25(0.4783-0 . \widehat{45})=0.006$. 
However, the longer is the time horizon for the agreement, the greater are the accumulated emissions within this period, and the less adequate is a responsibility concept exclusively based on emissions prior to the starting time. One possibility to overcome this drawback would be to define a timevarying concept of responsibility, evolving through time with current emissions. Alternatively, a flexible responsibility could be analyzed considering a sequence of short-horizon agreements with a changing initial responsibility. Either alternative is out of the scope of the paper.

Leaving aside the previous concern regarding responsibility, the proposed sharing mechanism can be extended to an infinite horizon. In the definition of the benefits from cooperation (8), the present value of the gap between the scrap values, $\left[D^{i}\left(P_{\mathrm{N}}(T ; t)\right)-D^{i}\left(P_{\mathrm{C}}(T)\right)\right] e^{-\rho(T-t)}$, vanishes when $T$ tends to infinity unless pollution explodes in the non-cooperative scenario. However, this definition, as well as the contributions in (9), are still valid when $T=\infty$. In consequence, as long as the surplus, $S(t)$, remains strictly positive and bounded, the surplus share, $\phi^{i}(t)$, and the relative contributions (equation (21) and (23) respectively) are valid like in the case of a finite horizon. Thus, Proposition 3 and its conclusions still applies.

In what follows, we characterize the effect of the length of the cooperative agreement in the particular example presented in the previous section. This particular example seeks to collect the idea that an environmental agreement implies current costs, in terms of lower emissions within cooperation, and future benefits from a cleaner environment. Thus $l_{N}^{i}(\tau ; t)=l_{C}^{i}(\tau)=0$ in (8). ${ }^{18}$ The numerical analysis carried out in Section 4 is recomputed for two other time horizons: $T=3$ and $T=4$. The comparison for the three cases is presented next.
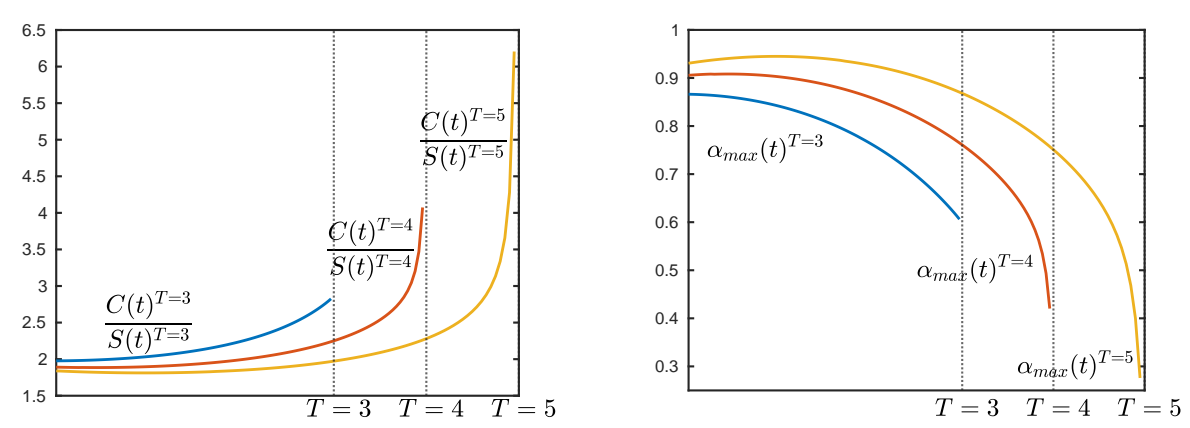

Fig. $7 C(t) / S(t)$ (left); and $\alpha_{\max }(t)$ (right).

Widening the horizon of the cooperative agreement, $T$, also widens the gap between the noncooperative and the cooperative pollution stock at the end of this period, and hence the gap $D^{i}\left(P_{\mathrm{N}}(T ; t)\right)-$ $D^{i}\left(P_{\mathrm{C}}(T)\right), \forall i \in\{1,2\}$, which defines the benefits from cooperation. As shown in Figure 7 (left), a longer cooperation shifts down the contributions per unit of surplus. The increment in the benefits from a

\footnotetext{
18 In the case of an infinite horizon, $T=\infty$, benefits, contributions and surplus would be equal to 0 . There would be no surplus to share. Indeed, in that case the Nash equilibrium matches the cooperative solution.
} 

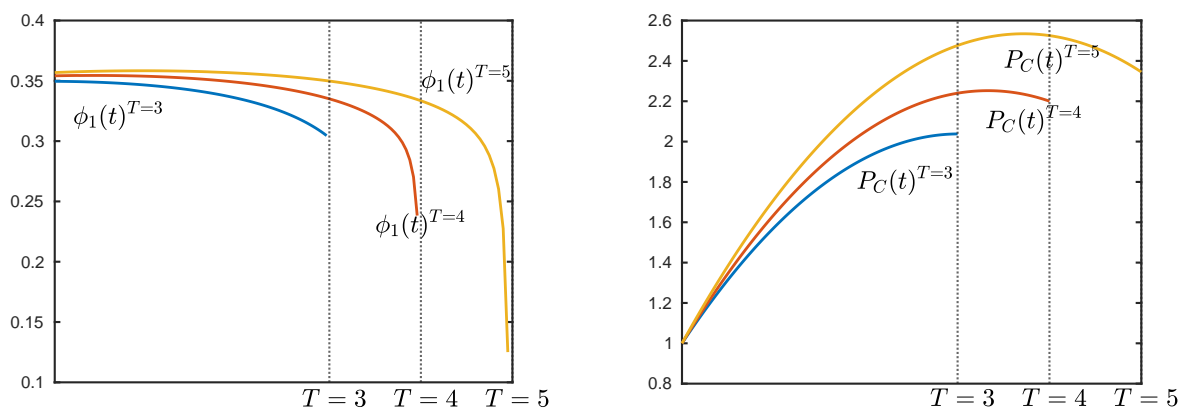

Fig. $8 \phi^{1}(t)$ (left); and $P_{\mathrm{C}}(t)$ (right).

longer cooperative agreement is relatively stronger than the increment in contributions:

$$
\frac{\partial B(t)}{\partial T} \frac{1}{B(t)}>\frac{\partial C(t)}{\partial T} \frac{1}{C(t)}
$$

Because $\hat{B}^{i}(t)=\hat{D}^{i}(P)$ is constant, then $\alpha_{\max }(t)$ is inversely related to the ratio of contributions per unit of surplus, as depicted in Figure 7 (right). Two conclusions stem from this figure regarding the maximum weight that the sharing mechanism can assign to responsibility. First, one observes that the curve $\alpha_{\max }(t)$ sifts up with the length of the cooperative agreement. This implies that the axiom of responsibility is satisfied, at least initially, for a wider rage of weighs. However, this figure also shows that $\alpha_{\max }(t)$ drops lower at the end of the cooperation, the longer is this interval. Therefore, if the responsibility axiom needs to be satisfied across the whole cooperative period, the longer this period, the less weight can be assigned to responsibility.

Figure 8 (left) depicts the ratio of the total surplus assigned to the responsible Region 1. Because the contribution per unit of surplus shifts down with the duration of cooperation, correspondingly, this region receives a greater ratio of the total surplus, and the opposite applies for the Region 2 (more severely hit by the environmental problem). Finally, Figure 8 (right) shows that a shorter cooperative agreement is characterized by a stronger effort in emissions reduction, leading to a slower increment in the pollution stock. Thus, short-term cooperative agreement can be more effective in environmental terms, although less efficient in terms of contribution per unit of benefit.

\section{Conclusions}

We analyze a finite-time cooperative agreement between two regions in order to carry out abatement activities in a stock pollution problem. We propose an imputation distribution procedure which defines how to share the efforts in the form of emissions reductions, while the situation being such that the environmental benefits from the agreement cannot be redistributed. This IDP is designed considering an asymmetric setting. The two parts differently value the environment and, in consequence, they are differently benefited by the agreement. Moreover, they are differently responsible for the accumulated emissions prior to the signing of the cooperative agreement. 
At any time while the two parts cooperate, the proposed sharing mechanism splits the total ongoing surplus from cooperation between the two regions. This sharing rule guarantees the time-consistency of the cooperative solution: each region prefers to remain in the agreement rather than to deviate to a non-cooperative mode of play from this time onward. The allocation rule is defined so that two additional desirable properties are satisfied. First, a benefits pay principle: ceteris paribus, the more one region benefits from the agreement, the higher its relative contribution. Second, a polluter pay principle: ceteris paribus, the more responsible a region is for past emissions, the greater its relative contribution.

The proposed sharing scheme contributes to the literature on dynamic distribution schemes on several aspects. First of all, it is not based on a specific cooperative solution concept (as is common in the literature). We seek a distribution scheme which jointly satisfies time consistency, the BPP, and the PPP, and we come up with a family of sharing rules which fulfill these properties. The IDP weighs each region's relative benefit from cooperation versus its responsibility for past emissions to determine its share of the total surplus to go.

If the responsible region is, at the same time, the least benefited by the agreement, then it is proven that the egalitarian rule or the symmetric NBS never fulfills the three desired properties. Interestingly, our proposed IDP is equivalent to an asymmetric NBS where the bargaining power was defined by the same function which, in our distribution scheme, divides the total surplus to go between the two regions. In consequence, if the regions' bargaining power were known, they would determine the weight given to responsibility in the proposed IDP. Conversely, if the bargaining power of each region is unknown, the proposed IDP is undetermined in the weight assigned to responsibility.

The proposed IDP is applied to a particular example under simplifying assumptions that maintain the essence of the problem: A linear-quadratic specification; current gains from production only dependent on emissions, and environmental losses only dependent on the stock of pollution (multiplicatively separable) and placed at the end of the cooperative period. Under these assumptions, the relative damage from pollution and the relative benefit from cooperation are equivalent. Thus, a higher relative benefit from the agreement corresponds to a higher relative damage from pollution and, in consequence, a lower net responsibility. If the weight given to responsibility is not too high, then the BPP is stronger than the PPP, which we defined as "strong-BPP".

The numerical example serves to illustrate previous findings. In particular, it analyzes the role played by the weight given to responsibility. As this weight gets bigger, the share of the surplus assigned to the responsible region shrinks and even vanishes. Correspondingly, its relative contribution increases. This weight determines whether a side-payment flows from the responsible to the non-responsible region (if large) or vice versa (if small). The numerical example also illustrates that a rise in one region's relative damage from pollution (i.e. its relative benefit from the agreement) rises its relative contribution. This strong-BPP is true only if the weight given to responsibility is lower than one. The numerical example 
also allows for a sensitivity analysis, in particular on the length of the cooperative agreement. It is observed that short-term cooperative agreements can be more effective in keeping pollution at bay than long-term cooperation. Accordingly, a stronger effort in terms of emission reductions, measured as the contribution per unit of benefit, is required. Moreover, this effort is more strongly borne by the responsible region.

An important concern regarding this, or any IDP involving side-payments, lies on its practical feasibility. In particular, on the practical feasibility of monetary transfers, specially international transfers. Some attempts have been implemented to facilitate them, like the REDD + UN program. And some literature supports ecological compensation, for example, water pollution compensation from downstream riparian regions to upstream regions. Nonetheless, the willingness of countries to accept side-payments, and hence the actual cost of these transfers, is an important issue that should be introduced in the analysis. Regarding this issue, we believe that transfers are less difficult and our IDP more realistic when dealing with more local environmental problems involving two (or in any case few) actors, rather than international problems with many countries.

Likewise, it is important to note that our analysis has considered only two regions. To generalize the proposed distribution procedure to more than two regions we should take into account two issues. The first issue is related to the implementation of the proposed scheme. We believe that the proposed definition, its characterization and the properties of the IDP remain valid in a more general setting with more agents. Nonetheless, a second important issue, related to the coalition stability under the proposed IDP should also be carefully studied. One possibility to generalize the analysis to several countries would be to consider two groups of countries, not necessarily of the same size. Within each group countries would be symmetric (but asymmetry remains between the two groups). The current analysis would be applied if it is further assumed that countries within a region collude to choose emissions even when, as a group, they behave non-cooperatively.

We also think that another interesting line of research would be to define responsibility as a function of time. This would help to implement the mechanism for a longer cooperative agreement, or even for an infinite time horizon.

\section{Data Availability Statement}

Data sharing not applicable to this article as no data sets were generated or analyzed during the current study. 


\section{Appendix A: Proof proposition 2, and remark 3}

Define by $S^{i}(t)$ the surplus to go from $t \in[0, T)$ on for any $i \in\{1,2\}$ under the proposed IDP. Then,

$$
W_{\pi}^{i}(t)=W_{\mathrm{N}}^{i}(t)+S_{\pi}^{i}(t), \quad \forall t \in[0, T) i \in\{1,2\}
$$

From the assumption of time consistency, $S_{\pi}^{i}(t) \geq 0$, for any $t \in[0, T)$ and any $i \in\{1,2\}$. Moreover, from condition (4) $W_{\pi}(t)=W_{\mathrm{C}}(t)$, and hence, $S(t)=S_{\pi}^{i}(t)+S_{\pi}^{-i}(t)$. Then, necessarily follows:

$$
S_{\pi}^{i}(t)=\phi^{i}(t) S(t), \quad \text { with } \phi^{i}(t) \geq 0 \text { and } \sum_{i=1}^{2} \phi^{i}(t)=1,
$$

which are conditions (16) and (17).

Next we prove that if the IDP verifies (16) and (17) then it is time consistent and verifies condition (4). Moreover we are going to explicitly find $\pi_{i}$ given in remark 3 . Computing the time derivatives in (16) we get: ${ }^{19}$

$$
\dot{W_{\pi}^{i}}=-\pi^{i}+\rho W_{\pi}^{i}, \quad \dot{W_{\pi}^{i}}=\dot{W}_{N}^{i}+\dot{\phi}^{i} S+\phi^{i} \dot{S}
$$

And computing the time derivatives in (7) and (6)

$$
\begin{gathered}
\dot{W}_{\mathrm{C}}^{i}=-w_{\mathrm{C}}^{i}+\rho W_{\mathrm{C}}^{i}, \\
\dot{W}_{\mathrm{N}}^{i}=-w_{\mathrm{N}}^{i}+\rho W_{\mathrm{N}}^{i}+\int_{t}^{T} \dot{w}_{\mathrm{N}}^{i}(\tau ; t) e^{-\rho(\tau-t)} d \tau-\left(D^{i}\right)^{\prime}\left(P_{\mathrm{N}}(T ; t)\right) \dot{P}_{\mathrm{N}}(T ; t) e^{-\rho(T-t)} .
\end{gathered}
$$

We call

$$
I_{\mathrm{N}}^{i}(t)=\int_{t}^{T} \dot{w}_{\mathrm{N}}^{i}(\tau ; t) e^{-\rho(\tau-t)} d \tau-\left(D^{i}\right)^{\prime}\left(P_{\mathrm{N}}(T ; t)\right) \dot{P}_{\mathrm{N}}(T ; t) e^{-\rho(T-t)} .
$$

Using these equations we get

$$
-\pi^{i}+\rho W_{\pi}^{i}=-w_{\mathrm{N}}^{i}+\rho W_{\mathrm{N}}^{i}+I_{\mathrm{N}}^{i}+\dot{\phi}^{i} S+\phi^{i} \dot{S}=-w_{\mathrm{N}}^{i}+\rho\left(W_{\pi}^{i}-\phi^{i} S\right)+I_{\mathrm{N}}^{i}+\dot{\phi}^{i} S+\phi^{i} \dot{S},
$$

then using that $\dot{S}=\dot{W}_{\mathrm{C}}^{i}+\dot{W}_{\mathrm{C}}^{-i}-\dot{W}_{\mathrm{N}}^{i}-\dot{W}_{\mathrm{N}}^{-i}$,

$$
\pi^{i}=w_{\mathrm{N}}^{i}+\rho \phi^{i} S-I_{\mathrm{N}}^{i}-\dot{\phi}^{i} S-\phi^{i} \dot{S}-\phi_{i}\left[\rho S+w_{\mathrm{N}}^{i}-w_{\mathrm{C}}^{i}-I_{\mathrm{N}}^{i}+w_{\mathrm{N}}^{-i}-w_{\mathrm{C}}^{-i}-I_{\mathrm{N}}^{-i}\right]
$$

Calling

$$
I V C^{i}(t)=w_{\mathrm{C}}^{i}(t)-w_{\mathrm{N}}^{i}(t)+\int_{t}^{T} \dot{w}_{\mathrm{N}}^{i}(\tau ; t) e^{-\rho(\tau-t)} d \tau-\left(D^{i}\right)^{\prime}\left(P_{\mathrm{N}}(T ; t)\right) \dot{P}_{\mathrm{N}}(T ; t) e^{-\rho(T-t)},
$$

and $I V C(t)=\sum_{i=1}^{2} I V C^{i}(t)$, we obtain the result. Moreover:

$$
\pi^{i}+\pi^{-i}=w_{\mathrm{C}}^{i}+w_{\mathrm{C}}^{-i}-I V C-\left(\dot{\phi}^{i}+\dot{\phi}^{-i}\right) S+\left(\phi^{i}+\phi^{-i}\right) I V C=w_{\mathrm{C}}^{i}+w_{\mathrm{C}}^{-i}
$$

\section{Appendix B: Cooperative and non-cooperative solutions to the L-Q differential game in}

\section{Section 4}

The solution to the cooperative problem (2) subject to (1) must satisfy the Hamilton-Jacobi-Bellman equation:

$$
\begin{aligned}
& \rho V^{C}(P, t)+\frac{\partial V^{C}(P, t)}{\partial t}=\max _{E^{1}, E^{2}}\left\{w^{1}\left(E^{1}\right)+w^{2}\left(E^{2}\right)+\frac{\partial V^{C}(P, t)}{\partial P}\left(E^{1}+E^{2}-\delta P\right)\right\}, \\
& \text { s.t.: } V(P(T), T)=-\left(d_{1}+d_{2}\right) P^{2}(T) .
\end{aligned}
$$

19 An upper dot refers to the derivative wrt $t$. For shortness and clarity we remove the time arguments in this proof when no confusion can arise. 
By conjecturing a linear quadratic value function, $V^{C}(P, t)=v_{2}^{C}(t) P^{2}+v_{1}^{C}(t) P+v_{0}^{C}(t)$, and taking into account the functional forms in (32), one gets the following system of 5 Riccati differential equations.

$$
\begin{aligned}
& \rho v_{2}^{C}(t)-\dot{v}_{2}(t)=-2\left(\delta-2 v_{2}^{C}(t)\right) v_{2}^{C}(t), \\
& \rho v_{1}^{C}(t)-\dot{v}_{1}(t)=-\delta v_{1}^{C}(t)+2\left(a_{1}+a_{2}+2 v_{1}^{C}(t)\right) v_{2}^{C}(t), \\
& \rho v_{0}^{C}(t)-\dot{v}_{0}(t)=1 / 2\left(a 1^{2}+a 2^{2}+2 v_{1}^{C}(t)\left(a 1+a 2+v_{1}^{C}(t)\right)\right), \\
& \dot{P}(t)=a_{1}+a_{2}-P(t) \delta+2 v_{1}^{C}(t)+4 P(t) v_{2}^{C}(t), \\
& P(0)=P_{0}, \quad v_{2}^{C}(T)=-\left(d_{1}+d_{2}\right), v_{1}^{C}(T)=v_{0}^{C}(T)=0 .
\end{aligned}
$$

The optimal expressions for $v_{2}^{C}(t) v_{1}^{C}(t), P^{C}(t)$ and $E_{i}^{C}(t)$ can be analytically computed from this system. We do not present them here for conciseness and because they do not add too much insight. They are available from the authors upon request.

Similarly, the non-cooperative optimization problem (3) subject to (1) must satisfy the Hamilton-Jacobi-Bellman equations:

$$
\begin{aligned}
& \rho V^{1 N}(P, t)+\frac{\partial V^{1 N}(P, t)}{\partial t}=\max _{E^{1}}\left\{w^{1}\left(E^{1}\right)+\frac{\partial V^{1 N}(P, t)}{\partial P}\left(E^{1}+E^{2}-\delta P\right)\right\}, \\
& \rho V^{2 N}(P, t)+\frac{\partial V^{2 N}(P, t)}{\partial t}=\max _{E^{2}}\left\{w^{2}\left(E^{2}\right)+\frac{\partial V^{2 N}(P, t)}{\partial P}\left(E^{1}+E^{2}-\delta P\right)\right\}, \\
& \text { s.t.: } V^{1 N}(P(T), T)=-d_{1} P^{2}(T), V^{2 N}(P(T), T)=-d_{2} P^{2}(T) .
\end{aligned}
$$

By again conjecturing linear quadratic value functions, $V^{i N}(P, t)=v_{2}^{i N}(t) P^{2}+v_{1}^{i N}(t) P+v_{0}^{i N}(t)$, for region $i \in\{1,2\}$, and taking into account the functional forms in (32), the following system of 7 Riccati differential equations must hold.

$$
\begin{aligned}
& \rho v_{2}^{1 N}(t)-\dot{v}_{2}^{1 N}(t)=2 v_{2}^{1 N}(t)\left(-\delta+v_{2}^{1 N}(t)+2 v_{2}^{2 N}(t)\right) \\
& \rho v_{2}^{2 N}(t)-\dot{v}_{2}^{2 N}(t)=2 v_{2}^{2 N}(t)\left(-\delta+2 v_{2}^{1 N}(t)+v_{2}^{2 N}(t)\right) \\
& \rho v_{1}^{1 N}(t)-\dot{v}_{1}^{1 N}(t)=2 v_{2}^{1 N}(t)\left(a_{1}+a_{2}+v_{1}^{2 N}(t)\right)+v_{1}^{1 N}(t)\left(-\delta+2 v_{2}^{1 N}(t)+2 v_{2}^{2 N}(t)\right) \\
& \rho v_{1}^{2 N}(t)-\dot{v}_{1}^{2 N}(t)=-\left(\delta-2 v_{2}^{1 N}(t)\right) v_{1}^{2 N}(t)+2\left(a_{1}+a_{2}+v_{1}^{1 N}(t)+v_{1}^{2 N}(t)\right) v_{2}^{2 N}(t) \\
& \rho v_{0}^{1 N}(t)-\dot{v}_{0}^{1 N}(t)=\frac{1}{2}\left(a_{1}^{2}+\left(v_{1}^{1 N}(t)\right)^{2}+2 v_{1}^{1 N}(t)\left(a_{1}+a_{2}+v_{1}^{2 N}(t)\right)\right) \\
& \rho v_{0}^{2 N}(t)-\dot{v}_{0}^{2 N}(t)=\frac{1}{2}\left(a_{2}^{2}+2\left(a_{1}+a_{2}+v_{1}^{1 N}(t)\right) v_{1}^{2 N}(t)+\left(v_{1}^{2 N}(t)\right)^{2}\right) \\
& \dot{P}(t)=a_{1}+a_{2}-P(t) \delta+v_{1}^{1 N}(t)+2 P v_{2}^{1 N}(t)+v_{1}^{2 N}(t)+2 P v_{2}^{2 N}(t), \\
& \text { s.t.: } P(0)=P_{0}, v_{2}^{1 N}(T)=-d_{1}, v_{2}^{2 N}(T)=-d_{2}, v_{1}^{1 N}(T)=v_{1}^{2 N}(T)=v_{0}^{1 N}(T)=v_{0}^{2 N}(T)=0 .
\end{aligned}
$$

The solution to this system cannot be analytically computed. 


\section{References}

1. Admati AR, Perry M (1991) Joint Projects without Commitment. Rev Econ Studies 58: 259-276

2. Barrett S (2013) Climate treaties and approaching catastrophes. J Environ Econ 66: 235-250. https://doi.org/10.1016/j.jeem.2012.12.004

3. Buchholz W, Peters W (2007) Justifying the Lindahl solution as an outcome of fair cooperation. Public Choice 133: 157-169. https://doi.org/10.1007/s11127-007-9184-7

4. Cabo F, Tidball M (2017) Promotion of cooperation when benefits come in the future: A water transfer case. Resour Energy Econ 47: 56-71. https://doi.org/10.1016/j.reseneeco.2016.12.001.

5. Cabo F, Escudero E, Martín-Herrán G (2006) A time-consistent agreement in an interregional differential game on pollution and trade. Int Game Theory Rev 8: 369-393. https://doi.org/10.1142/S0219198906000977

6. De Villemeur EB, Leroux J (2011) Sharing the cost of Global Warming. Scand J Econ 113: 758-83. www.jstor.org/stable/41407744

7. Fanokoa PS, Telahigue I, Zaccour G (2011) Buying cooperation in an asymmetric environmental differential game. J Econ Dyn Control 35: 935-946. https://doi.org/10.1016/j.jedc.2010.11.008.

8. Fershtman C, Nitzan S (1991) Dynamic voluntary provision of public goods. Europ Econ Rev 35: 1057-1067

9. Guttman J (1978) Understanding collective action: Matching behavior. Amer Econ Rev Association Papers and Proceedings 68: 251-255

10. Guttman J (1987) A non-Cournot model of voluntary collective action. Economica 54: 1-19

11. Hayner M, Weisbach D (2016) Two theories of responsibility for past emissions. Midwest Stud Philos 40: 96-113. doi:10.1111/misp.12049

12. Jørgensen S, Zaccour G (2001) Time consistent side payments in a dynamic game of downstream pollution. J Econ Dyn Control 25: 1973-1987. https://doi.org/10.1016/S0165-1889(00)00013-0

13. Jørgensen S, Martín-Herrán, G, Zaccour G (2003) Agreeability and Time-Consistency in Linear-State Differential Games. J Optimiz Theory App 119: 49-63

14. Jørgensen S, Martín-Herrán, G, Zaccour G (2005) Sustainability of cooperation overtime in Linear-Quadratic Differential Games. Int Game Theory Rev 7: 395-406

15. Kaitala V, Pohjola M. (1990) Economic development and agreeable redistribution in capitalism: Efficient Game Equilibria in a two-class Neoclassical Growth Model. Int Econ Rev 31:421-437

16. Petrosyan LA, Danilov NN (1979) Stable solutions in Differential Games with transferable payoffs (in Russian). Viestnik of the Leningrad State University. 1979 N1, 52-59

17. Petrosyan LA (1993) Differential Games of Pursuit. World Scientific, Singapour.

18. Petrosyan LA, Zaccour G (2003) Time-consistent Shapley value allocation of pollution cost reduction. J Econ Dyn Control 27: 381-398. https://doi.org/10.1016/S0165-1889(01)00053-7

19. Pigou AC (1920) The Economics of Welfare. Macmillan, London.

20. Singer P (2004) One World: The Ethics of Globalization. Yale University Press, New Haven, CT.

21. Sorger G (2006) Recursive bargaining over a productive asset. J Econ Dyn Control 30: 2637-2659. https://doi.org/10.1016/j.jedc.2005.08.005

22. Ward DS, Mahowald NM (2014) Contributions of developed and developing countries to global climate forcing and surface temperature change. Environ Res Lett 9: 074008. http://dx.doi.org/10.1088/1748-9326/9/7/074008

23. Wei T, Dong W, Yan Q, Chou J, Yang Z, Tian T (2016) Developed and developing world contributions to climate system change based on carbon dioxide, methane and nitrous oxide emissions. Adv Atmos Sci 33: 632-643. https://doi.org/10.1007/s00376-015-5141-4

24. Wicksell K (1896/1958) Ein neues Prinzip der gerechten Besteuerung. Finanztheoretische Untersuchungen. Jena: Gustav Fischer; partially translated as: A new principle of just taxation. In: Musgrave RA, Peacock AT (eds) Classics in the theory of public finance. St. Martin's Press, New York, pp 72-118 
25. Wirl, F (1996) Dynamic voluntary provision of public goods: Extension to nonlinear strategies. Eur J Political Econ 12: 555-560. https://doi.org/10.1016/S0176-2680(96)00016-X

26. Yeung DW, Petrosyan L (2018) Dynamic Shapley Value and Dynamic Nash Bargaining. Nova Science, NewYork.

27. Zaccour G (2008) Time consistency in cooperative Differential Games: A tutorial. INFOR 46: 81-92. doi: 10.3138/infor.46.1.81

28. Zhang ZQ, Qu JS, Zeng JJ (2008) A quantitative comparison and analytical study on the assessment indicators of greenhouse gases emissions. J Geogr Sci 18: 387-399. doi: 10.1007/s11442-008-0387-8. 